\title{
ON THE QUANTUM LORENTZ GROUP
}

\author{
M. Lagraa \\ Laboratoire de Physique Théorique \\ Université d'Oran Es-Sénia, 31100, Algérie \\ and \\ Laboratoire de Physique Théorique et Hautes énergies\$, Batiment 211, \\ Université Paris XI, 94405 ORSAY,France
}

\begin{abstract}
The quantum analogues of Pauli matrices are introduced and investigated. From these matrices and an appropriate trace over spinorial indices we construct a quantum Minkowsky metric. In this framwork we show explicitly the correspondence between the $S L(2, C)$ and Lorentz quantum groups. The $\mathcal{R}$ matrices of the quantum Lorentz group are constructed in terms of the $\mathrm{R}$ matrices of $S L(2, C)$ group. These $\mathcal{R}$ matrices satisfy adequate properties as Yang-Baxter equations, Hecke relations and quantum symmetrization of the metric. It is also shown that the Minkowsky metric leads to an invariant and central norm.
\end{abstract}

\footnotetext{
${ }^{1}$ Laboratoire associé au Centre National de la Recherche Scientifique - URA D0063
} 


\section{Introduction}

The Lorentz group plays a fundamental role in physics. First, it constitutes the homogeneous part of Poincaré group which is intrinsically connected to the geometry of the space-time and leaves invariant all physical systems discribed by the special theory of relativity. Second, the different representations of Lorentz group are field discribing particles which constitute the physical systems. For these reasons, it is especially interesting to study the noncommutative version of the Lorentz group.

The other reason which makes the study of the quantum Lorentz group interesting is due to the fact that in quantum field theory based on a classical space-time and a classical lorentz group there exist difficulties tied to small space-time distances. One may hope to solve these difficulties by new tools provided by the noncommutative geometry [1-4].

The construction of quantum Lorentz group has been considered by many authors, either directly [5-6] or in the context of the quantum poincaré group [7-8]. Despite intensive efforts, these studies do not completly describe the quantum Lorentz group.

In this paper one constructs quantum Lorentz group out of the quantum $S L(2, C)$ group by showing how all properties of the former can be deduced from the later which are well known. The paper is organized in the following way. In Sect. 2 we recall the well known results provided by the bicovariant calculus over $S L(2, C)$ and $S U(2)$ quantum groups. We shall assume that the undotted (conjugate) and dotted generators of quantum $S L(2, C)$ group satisfy the commutation rules of the quantum $S U(2)$ group.

In Sect. 3 the construction of quantum Lorentz group is carried out of the quantum $S L(2, C)$ group following the analogue of the homomorphism for the classical group $S O(1,3) \sim S L(2, C) \backslash Z_{2}$. We shall start by investigating the quantum analogues of the Pauli matrices from which we construct an adequate Minkowsky metric. An example of a two parameters deformation is given and the completeness relations are established. From the properties of the quantum Pauli matrices and the generators of $S L(2, C)$, we construct the generators of the quantum Lorentz group. We show that they satisfy the axiomatic structure of the Hopf algebras and the orthogonality relations. We also construct the $\mathcal{R}$ matrices of the Lorentz group out of those of $S L(2, C)$ group. These $\mathcal{R}$ matrices satisfy the Yang-Baxter equations, the Hecke relations and exhibit the quantum symetrization properties of the Minkowsky metric.

In Sect. 4 we investigate the properties of the Minkowsky space. In particular, we show that the Minkowsky metric induces an invariant norm which commutes with the Hopf algebra $\mathcal{A}$ generated by the quantum $S L(2, C)$ group generators, the undotted and dotted basis (spinors) of the bicovariant bimodule over $\mathcal{A}$ and the quantum coordinates of the Minkowsky space.

\section{Bicovariant Calculus On $S L(2, C)$ and $S U(2)$ Quantum Groups}

Before we start to construct the quantum Lorentz group out of the quantum $S L(2, C)$ group, let us recall some results provided by the bicovariant calculus over the $S L(2, C)$ and $S U(2)$ 
quantum groups. Let an unital $\star$-algebra $\mathcal{A}$ generated by $M_{\alpha}{ }^{\beta}(\alpha, \beta=1,2)$ which preserves a nondegenerate bilinear form $\varepsilon$

$$
\varepsilon_{\alpha \beta} M_{\gamma}^{\alpha} M_{\delta}^{\beta}=\varepsilon_{\gamma \delta} I_{\mathcal{A}} \quad, \quad \varepsilon^{\gamma \delta} M_{\gamma}^{\alpha} M_{\delta}{ }^{\beta}=\varepsilon^{\alpha \beta} I_{\mathcal{A}} \quad, \quad \varepsilon^{\alpha \gamma} \varepsilon_{\gamma \beta}=\delta_{\beta}^{\alpha}=\varepsilon_{\beta \gamma} \varepsilon^{\gamma \alpha}
$$

which are the unimodularity conditions. The nondegenerate bilinear form $\varepsilon$ is considered as a quantum spinor metric, $I_{\mathcal{A}}$ being the unity of $\mathcal{A}$. To preserve these conditions under the antimultiplicative involution $\star: \mathcal{A} \rightarrow \mathcal{A}$, the spinor metric must satisfy the condition $\left(\varepsilon_{\alpha \beta}\right)^{\star}=\lambda \varepsilon_{\dot{\beta} \dot{\alpha}}$ with $\lambda \lambda^{\star}=1$ leading to:

$$
\varepsilon_{\dot{\alpha} \dot{\beta}} M_{\dot{\gamma}}^{\dot{\alpha}} M_{\dot{\delta}}^{\dot{\beta}}=\varepsilon_{\dot{\gamma} \dot{\delta}} I_{\mathcal{A}} \quad, \quad \varepsilon^{\dot{\gamma} \dot{\delta}} M_{\dot{\gamma}}^{\dot{\alpha}} M_{\dot{\delta}}^{\dot{\beta}}=\varepsilon^{\dot{\alpha} \dot{\beta}} I_{\mathcal{A}} \quad, \quad \varepsilon^{\dot{\alpha} \dot{\gamma}} \varepsilon_{\dot{\gamma} \dot{\beta}}=\delta_{\dot{\beta}}^{\dot{\alpha}}=\varepsilon_{\dot{\beta} \dot{\gamma}} \varepsilon^{\dot{\gamma} \dot{\alpha}}
$$

where $M_{\dot{\alpha}}^{\dot{\beta}}=\left(M_{\alpha}{ }^{\beta}\right)^{\star}$. For convenience, we take $\lambda=1$. $\mathcal{A}$ carries a structure of a $\star$-Hopf algebra with a coaction $\Delta: \mathcal{A} \rightarrow \mathcal{A} \otimes \mathcal{A}$, a counit $\varepsilon: \mathcal{A} \rightarrow \mathcal{C}$ and an antipode $S: \mathcal{A} \rightarrow \mathcal{A}$ defined on the generators by $\Delta\left(M_{\alpha}^{\beta}\right)=M_{\alpha}^{\gamma} \otimes M_{\gamma}{ }^{\beta}, \varepsilon\left(M_{\alpha}^{\beta}\right)=\delta_{\alpha}^{\beta}$ and $S\left(M_{\alpha}^{\beta}\right)=\varepsilon_{\alpha \gamma} M_{\delta}{ }^{\gamma} \varepsilon^{\delta \beta}$. On the dotted copy, we have $\left(\Delta\left(M_{\alpha}{ }^{\beta}\right)\right)^{\star}=\Delta\left(\left(M_{\alpha}{ }^{\beta}\right)^{\star}\right)=\Delta\left(M_{\dot{\alpha}}^{\dot{\beta}}\right)=M_{\dot{\alpha}}^{\dot{\gamma}} \otimes M_{\dot{\gamma}}^{\dot{\beta}}, \varepsilon\left(M_{\dot{\alpha}}^{\dot{\beta}}\right)=\delta_{\dot{\alpha}}^{\dot{\beta}}$ and $S\left(M_{\dot{\alpha}}^{\dot{\beta}}\right)=\varepsilon_{\dot{\alpha} \dot{\gamma}} M_{\dot{\delta}}^{\dot{\gamma}} \varepsilon^{\dot{\delta} \dot{\beta}}$. the involution $\star$ acts on the antipode as $\left(S\left(M_{\alpha}^{\beta}\right)\right)^{\star}=\varepsilon^{\dot{\beta} \dot{\delta}} M_{\dot{\delta}}^{\dot{\gamma}} \varepsilon_{\dot{\gamma} \dot{\alpha}}=$ $S^{-1}\left(M_{\dot{\alpha}}^{\dot{\beta}}\right)$.

It is known [9] that the generators of a such system satify the noncommutativity relations $R_{\sigma \rho}^{ \pm \alpha \beta} M_{\gamma}{ }^{\sigma} M_{\delta}{ }^{\rho}=M_{\sigma}{ }^{\alpha} M_{\rho}{ }^{\beta} R_{\gamma \delta}^{ \pm \sigma \rho}$ where the forms of the $\mathrm{R}$ matrices are given by $R_{\gamma \delta}^{ \pm \alpha \beta}=$ $\delta_{\gamma}^{\alpha} \delta_{\delta}^{\beta}+a^{ \pm 1} \varepsilon^{\alpha \beta} \varepsilon_{\gamma \delta}$ satifying $R_{\sigma \rho}^{ \pm \alpha \beta} R_{\gamma \delta}^{\mp \sigma \rho}=\delta_{\gamma}^{\alpha} \delta_{\delta}^{\beta}$ with $a+a^{-1}+\varepsilon^{\alpha \beta} \varepsilon_{\alpha \beta}=0$, and $a \neq 0$. These $\mathrm{R}$ matrices satisfy the Yang-Baxter equation, the Hecke equations $\left(R^{ \pm}+a^{ \pm 2}\right)\left(R^{ \pm}-1\right)$ and $\varepsilon_{\alpha \beta} R_{\sigma \gamma}^{ \pm \alpha \lambda} R_{\lambda \delta}^{ \pm \beta \rho}=a^{\mp 1} \varepsilon_{\gamma \delta} \delta_{\sigma}^{\rho}$.

Now, we consider a right-invariant basis $\theta_{\alpha}$ of the bicovariant bimodule $\Gamma$ over $\mathcal{A}$ on which the right coaction acts as $\Delta_{R}\left(\theta_{\alpha}\right)=\theta_{\alpha} \otimes I, \Delta_{R}\left(\theta_{\dot{\alpha}}\right)=\theta_{\dot{\alpha}} \otimes I$ and the left coaction acts as

$$
\begin{aligned}
& \Delta_{L}\left(\theta_{\alpha}\right)=M_{\alpha}^{\beta} \otimes \theta_{\beta} \quad, \quad \Delta_{L}\left(\theta^{\alpha}\right)=S\left(M_{\beta}^{\alpha}\right) \otimes \theta^{\beta} \\
& \Delta_{L}\left(\theta_{\dot{\alpha}}\right)=M_{\dot{\alpha}}^{\dot{\beta}} \otimes \theta_{\dot{\beta}} \quad, \quad \Delta_{L}\left(\theta^{\dot{\alpha}}\right)=S^{-1}\left(M_{\dot{\beta}}^{\dot{\alpha}}\right) \otimes \theta^{\dot{\beta}}
\end{aligned}
$$

where $\left(\theta_{\alpha}\right)^{\star}=\theta_{\dot{\alpha}}$ and the spinorial indices are lowered and raised as $\theta_{\alpha}=\theta^{\beta} \varepsilon_{\beta \alpha}, \theta^{\alpha}=\theta_{\beta} \varepsilon^{\beta \alpha}$, $\theta^{\dot{\alpha}}=\varepsilon^{\dot{\alpha} \dot{\beta}} \theta_{\dot{\beta}}$ and $\theta_{\dot{\alpha}}=\varepsilon_{\dot{\alpha} \dot{\beta}} \theta^{\dot{\beta}}$. From the bicovariance properties of the bimodule $\mathcal{A}-\Gamma[4]$ we can show the existence of functionals $f: \mathcal{A} \rightarrow C$ satisfying the following properties

$$
\begin{array}{rll}
\theta_{\alpha} a=\left(a \star f_{\alpha}{ }^{\beta}\right) \theta_{\beta} \quad & \theta^{\alpha} a=\left(a \star \tilde{f}_{\beta}^{\alpha}\right) \theta^{\beta} \\
\theta_{\dot{\alpha}} a=\left(a \star f_{\dot{\alpha}}^{\dot{\beta}}\right) \theta_{\dot{\beta}} \quad, & \theta^{\dot{\alpha}} a=\left(a \star \tilde{f}_{\dot{\beta}}^{\dot{\alpha}}\right) \theta^{\dot{\beta}} \\
a \theta_{\alpha}=\theta_{\beta}\left(a \star f_{\alpha}^{\beta} \circ S\right) \quad, \quad a \theta^{\alpha}=\theta^{\beta}\left(a \star \tilde{f}_{\beta}^{\alpha} \circ S\right) \\
a \theta_{\dot{\alpha}}=\theta_{\dot{\beta}}\left(a \star f_{\dot{\alpha}}{ }^{\dot{\beta}} \circ S\right) \quad, \quad & a \theta^{\dot{\alpha}}=\theta^{\dot{\beta}}\left(a \star \tilde{f}_{\dot{\beta}}^{\dot{\alpha}} \circ S\right) \\
f_{\alpha}{ }^{\beta}(a b)=f_{\alpha}^{\gamma}(a) f_{\gamma}{ }^{\beta}(b) \quad, & \tilde{f}_{\alpha}^{\beta}(a b)=\tilde{f}_{\gamma}^{\beta}(a) \tilde{f}_{\alpha}^{\gamma}(b),
\end{array}
$$




$$
\begin{aligned}
& f_{\dot{\alpha}}^{\dot{\beta}}(a b)=f_{\dot{\alpha}}^{\dot{\gamma}}(a) f_{\dot{\gamma}}^{\dot{\beta}}(b) \quad, \quad \tilde{f}_{\dot{\alpha}}^{\dot{\beta}}(a b)=\tilde{f}_{\dot{\gamma}}^{\dot{\beta}}(a) \tilde{f}_{\dot{\alpha}}^{\dot{\gamma}}(b) \\
& f_{\alpha}^{\beta}(I)=\delta_{\alpha}^{\beta}=\tilde{f}_{\alpha}^{\beta}(I) \quad, \quad f_{\dot{\alpha}}^{\dot{\beta}}(I)=\delta_{\dot{\alpha}}^{\dot{\beta}}=\tilde{f}_{\dot{\alpha}}^{\dot{\beta}}(I) \\
& M_{\alpha}^{\gamma}\left(f_{\gamma}^{\beta} \star a\right)=\left(a \star f_{\alpha}^{\gamma}\right) M_{\gamma}{ }^{\beta} \quad, \quad S\left(M_{\gamma}{ }^{\alpha}\right)\left(\tilde{f}_{\beta}^{\gamma} \star a\right)=\left(a \star \tilde{f}_{\gamma}{ }^{\alpha}\right) S\left(M_{\beta}{ }^{\gamma}\right) \text {, } \\
& M_{\dot{\alpha}}^{\dot{\gamma}}\left(f_{\dot{\gamma}}^{\dot{\beta}} \star a\right)=\left(a \star f_{\dot{\alpha}}^{\dot{\gamma}}\right) M_{\dot{\gamma}}^{\dot{\beta}} \quad, \quad S^{-1}\left(M_{\dot{\gamma}}^{\dot{\alpha}}\right)\left(\tilde{f}_{\dot{\beta}}^{\dot{\gamma}} \star a\right)=\left(a \star \tilde{f}_{\dot{\gamma}}^{\dot{\alpha}}\right) S^{-1}\left(M_{\dot{\beta}}^{\dot{\gamma}}\right)
\end{aligned}
$$

where the convolution product is defined by $a \star f=(f \otimes I) \Delta(a)$ for any $a \in \mathcal{A}$. Setting $a=S(a)\left(S^{-1}(a)\right)$ into the right undotted (dotted) relation of (9)((10)), then applying $S^{-1}(S)$ and comparing with the corresponding left undotted (dotted) relation of (9) $((10))$, we get

$$
f_{\alpha}^{\beta}=\tilde{f}_{\alpha}^{\beta} \circ S, f_{\dot{\alpha}}^{\dot{\beta}}=\tilde{f}_{\dot{\alpha}}^{\dot{\beta}} \circ S^{-1} .
$$

For the generators of $\mathcal{A}$, the left relation (9) gives $M_{\alpha}^{\gamma} M_{\rho}{ }^{\delta} f_{\gamma}{ }^{\beta}\left(M_{\delta}{ }^{\sigma}\right)=f_{\alpha}{ }^{\gamma}\left(M_{\rho}{ }^{\delta}\right) M_{\delta}{ }^{\sigma} M_{\gamma}{ }^{\beta}$ which shows that there exist two functionals $f_{ \pm \gamma}{ }^{\alpha}\left(M_{\delta}{ }^{\rho}\right)$ proportional to the $R_{\gamma \delta}^{ \pm \rho \alpha}$ matrices. Applying these functionals on both sides of the unimodularity condition, we obtain: $f_{ \pm \gamma}{ }^{\alpha}\left(M_{\delta}{ }^{\rho}\right)=a^{\mp \frac{1}{2}} R_{\gamma \delta}^{ \pm \rho \alpha}$ and $f_{ \pm \gamma}{ }^{\alpha}\left(S\left(M_{\delta}{ }^{\rho}\right)\right)=a^{ \pm \frac{1}{2}} R_{\delta \gamma}^{\mp \alpha \gamma}[10]$. The same procedure can be used for the dotted copy of $\mathcal{A}$ generators. Then there exist two basis $\theta_{ \pm \alpha}$ corresponding to the functionals $f_{ \pm \alpha}^{\beta}$.

Applying the $\star$ involution on both sides of (2) and (3), we get respectively

$$
\begin{gathered}
\left(\theta_{ \pm \alpha} a\right)^{\star}=\left(\theta_{ \pm \beta}\right)^{\star}\left(f_{ \pm \alpha}^{\beta}\left(a_{(1)}\right)\right)^{\star} a_{(2)}^{\star}=a^{\star}\left(\theta_{ \pm \alpha}\right)^{\star}, \quad\left(\theta_{ \pm}^{\alpha} a\right)^{\star}=\left(\theta_{ \pm}^{\beta}\right)^{\star}\left(\tilde{f}_{ \pm \beta}^{\alpha}\left(a_{(1)}\right)\right)^{\star} a_{(2)}^{\star}=a^{\star}\left(\theta_{ \pm}^{\alpha}\right)^{\star}, \\
\left(\theta_{ \pm \dot{\alpha}} a\right)^{\star}=\left(\theta_{ \pm \dot{\beta}}\right)^{\star}\left(f_{ \pm \dot{\alpha}}^{\dot{\beta}}\left(a_{(1)}\right)\right)^{\star} a_{(2)}^{\star}=a^{\star}\left(\theta_{ \pm \dot{\alpha}}\right)^{\star} \text { and }\left(\theta_{ \pm}^{\dot{\alpha}} a\right)^{\star}=\left(\theta_{ \pm}^{\dot{\beta}}\right)^{\star}\left(\tilde{f}_{ \pm \dot{\beta}}^{\dot{\alpha}}\left(a_{(1)}\right)\right)^{\star} a_{(2)}^{\star}=a^{\star}\left(\theta_{ \pm}^{\dot{\alpha}}\right)^{\star}(12)
\end{gathered}
$$

where $a_{(1)}$ and $a_{(2)}$ denote elements of $\Delta(a)=a_{(1)} \otimes a_{(2)}$. In the other hand, for $a=a^{\star}$, (4) and (5) give respectively

$$
\begin{array}{cc}
a^{\star} \theta_{ \pm \alpha}=\theta_{ \pm \beta} f_{ \pm \alpha}^{\beta}\left(S\left(a_{(1)}^{\star}\right)\right) a_{(2)}^{\star}, & a^{\star} \theta_{ \pm}^{\alpha}=\theta_{ \pm}^{\beta} \tilde{f}_{ \pm \beta}^{\alpha}\left(S\left(a_{(1)}^{\star}\right)\right) a_{(2)}^{\star} \\
a^{\star} \theta_{ \pm \dot{\alpha}}=\theta_{ \pm \dot{\beta}} f_{ \pm \dot{\alpha}} \dot{\dot{\beta}}\left(S\left(a_{(1)}^{\star}\right)\right) a_{(2)}^{\star} \quad, & a^{\star} \theta_{ \pm}^{\dot{\alpha}}=\theta_{ \pm}^{\dot{\beta}} \tilde{f}_{ \pm \dot{\beta}}^{\dot{\alpha}}\left(S\left(a_{(1)}^{\star}\right)\right) a_{(2)}^{\star} .
\end{array}
$$

But for $a=M_{\sigma}{ }^{\rho}$, we have $\left(f_{ \pm \alpha}^{\beta}\left(M_{\sigma}^{\delta}\right)\right)^{\star}=\left(a^{\mp \frac{1}{2}} R_{\alpha \sigma}^{ \pm \delta \beta}\right)^{\star}=a^{\mp \frac{1}{2}} R_{\dot{\sigma} \dot{\alpha}}^{ \pm \dot{\delta}}=f_{\mp \dot{\alpha}}^{\dot{\beta}}\left(S\left(M_{\dot{\sigma}}^{\dot{\delta}}\right)\right)$ for $a$ real. Therefore (12) and (13) are consistent if $\left(\theta_{ \pm \alpha}\right)^{\star}=\theta_{\mp \dot{\alpha}}$ and $\left(\theta_{ \pm}^{\alpha}\right)^{\star}=\theta_{\mp}^{\dot{\alpha}}$ yielding

$$
\begin{gathered}
\left(f_{ \pm \alpha}^{\beta}(a)\right)^{\star}=f_{\mp \dot{\alpha}}^{\dot{\beta}}\left(S\left(a^{\star}\right)\right),\left(\tilde{f}_{ \pm \alpha}^{\beta}(a)\right)^{\star}=\tilde{f}_{\mp \dot{\alpha}}^{\dot{\beta}}\left(S\left(a^{\star}\right)\right), \\
\left(f_{ \pm \dot{\alpha}}^{\dot{\beta}}(a)\right)^{\star}=f_{\mp \alpha}^{\beta}\left(S\left(a^{\star}\right)\right) \text { and }\left(\tilde{f}_{ \pm \dot{\alpha}}^{\dot{\beta}}(a)\right)^{\star}=\tilde{f}_{\mp \alpha}^{\beta}\left(S\left(a^{\star}\right)\right) .
\end{gathered}
$$

Finally, by using the spinor metric to raise the indices of the right invariant basis into (2) and (3), we may also show that

$$
f_{ \pm \alpha}^{\beta}=\varepsilon^{\beta \delta} \tilde{f}_{ \pm \delta}^{\gamma} \varepsilon_{\gamma \alpha} \text { and } \varepsilon^{\dot{\beta} \dot{\delta}} f_{ \pm \dot{\delta}} \dot{\gamma} \varepsilon_{\dot{\gamma} \dot{\alpha}}=\tilde{f}_{ \pm \dot{\alpha}}^{\dot{\beta}} .
$$

In this stage, we have no indication on the explicit forms of $f_{ \pm \gamma}^{\alpha}\left(M_{\dot{\delta}}{ }^{\dot{\rho}}\right)$ or $f_{ \pm \dot{\gamma}}^{\dot{\alpha}}\left(M_{\delta}{ }^{\rho}\right)$ to control the noncommutativity between undotted and dotted generators of the quantum $S L(2, C)$ group. 
To carry this point we assume either the generators $M_{\alpha}{ }^{\beta}$ commute with $M_{\dot{\alpha}}^{\dot{\beta}}$ or are controled by the $\mathrm{R}$ matrices satisfying the properties of the quantum $S U(2)$ group. In the following we assume the later possibility.

To reflect the specific properties of the quantum $S U(2)$ group, we have to add the unitarity condition on the generators as $M_{\dot{\alpha}}^{\dot{\beta}}=S\left(M_{\beta}{ }^{\alpha}\right)$ and $M_{\alpha}{ }^{\beta}=S^{-1}\left(M_{\dot{\beta}}^{\dot{\alpha}}\right)$. Applying $S$ on both sides of the unimodularity condition, we may show that the unitarity condition yields $\varepsilon_{\alpha \beta}=\lambda \varepsilon^{\dot{\beta} \dot{\alpha}}$ with $\lambda \lambda^{\star}=1$. In the following we take $\lambda=-1$. Then to be consistent with the quantum $S U(2)$ group, the spinor metric must satisfy

$$
\left(\varepsilon_{\alpha \beta}\right)^{\star}=\varepsilon_{\dot{\beta} \dot{\alpha}}=-\varepsilon^{\alpha \beta} \text { and }\left(\varepsilon^{\alpha \beta}\right)^{\star}=\varepsilon^{\dot{\beta} \dot{\alpha}}=-\varepsilon_{\alpha \beta} .
$$

It is easy to see, by using the unitarity condition of the generators into (1), that $\theta_{\dot{\alpha}}=\theta^{\alpha}$ and $\theta_{\alpha}=\theta^{\dot{\alpha}}$ implying conditions on the functionals

$$
f_{ \pm \dot{\alpha}}^{\dot{\beta}}=\tilde{f}_{ \pm \beta}^{\alpha} \quad, \quad \tilde{f}_{ \pm \dot{\alpha}}^{\dot{\beta}}=f_{ \pm \beta}^{\alpha}
$$

As stated above, we assume that the functionals of the quantum $S L(2, C)$ group satisfy the same properties as the $S U(2)$ ones. Therefore, if for example we set $a=M_{\dot{\sigma}}^{\dot{\rho}}$ into the left relation of $(2)$, we get

$$
M_{\alpha}^{\gamma} M_{\dot{\sigma}}^{\dot{\delta}} f_{ \pm \gamma}^{\beta}\left(M_{\dot{\delta}}^{\dot{\rho}}\right)=f_{ \pm \alpha}^{\gamma}\left(M_{\dot{\sigma}}^{\dot{\delta}}\right) M_{\dot{\delta}}^{\dot{\rho}} M_{\gamma}^{\beta} \quad \text { or } \quad M_{\alpha}^{\gamma} M_{\dot{\sigma}}^{\dot{\delta}} R_{\gamma \dot{\delta}}^{ \pm \dot{\rho} \beta}=R_{\alpha \dot{\sigma}}^{ \pm \dot{\delta} \gamma} M_{\dot{\delta}}^{\dot{\rho}} M_{\gamma}{ }^{\beta}
$$

where $f_{ \pm \alpha}^{\gamma}\left(M_{\dot{\sigma}}^{\dot{\delta}}\right)=R_{\alpha \dot{\sigma}}^{ \pm \dot{\delta} \gamma}=f_{ \pm \alpha}^{\gamma}\left(S\left(M_{\delta}{ }^{\sigma}\right)\right)=a^{ \pm \frac{1}{2}} R_{\delta \alpha}^{\mp \gamma \sigma}$. we have also objects of the form

$$
\begin{array}{r}
f_{ \pm \alpha}^{\gamma}\left(S\left(M_{\dot{\sigma}}^{\dot{\delta}}\right)\right)=R_{\dot{\sigma} \alpha}^{\mp \gamma \dot{\delta}}=f_{ \pm \alpha}^{\gamma}\left(S\left(S\left(M_{\delta}^{\sigma}\right)\right)\right)=\varepsilon_{\delta \lambda} f_{ \pm \alpha}^{\gamma}\left(S\left(M_{\nu}^{\lambda}\right)\right) \varepsilon^{\nu \sigma}= \\
a^{ \pm \frac{1}{2}} \varepsilon_{\delta \lambda} R_{\nu \alpha}^{\mp \gamma \lambda} \varepsilon^{\nu \sigma}=\varepsilon_{\dot{\sigma} \dot{\rho}} f_{ \pm \alpha}^{\gamma}\left(M_{\dot{\gamma}}^{\dot{\rho}}\right) \varepsilon^{\dot{\gamma} \dot{\delta}}=\varepsilon_{\dot{\sigma} \dot{\rho}} R_{\alpha \dot{\lambda}}^{ \pm \dot{\rho} \gamma} \varepsilon^{\dot{\lambda} \dot{\delta}}
\end{array}
$$

From (6) and (7), we get respectively

$$
\begin{aligned}
& f_{ \pm \alpha}^{\gamma}\left(M_{\dot{\sigma}}^{\dot{\delta}}\right) f_{ \pm \gamma}^{\beta}\left(S\left(M_{\dot{\delta}}^{\dot{\rho}}\right)\right)=\delta_{\alpha}^{\beta} \delta_{\dot{\sigma}}^{\dot{\rho}}=R_{\alpha \dot{\sigma}}^{ \pm \dot{\delta} \gamma} R_{\dot{\delta} \gamma}^{\mp \beta \dot{\rho}} \\
& f_{ \pm \alpha}^{\gamma}\left(S\left(M_{\dot{\sigma}}^{\dot{\delta}}\right)\right) f_{ \pm \gamma}^{\beta}\left(M_{\dot{\delta}}^{\dot{\rho}}\right)=\delta_{\alpha}^{\beta} \delta_{\dot{\sigma}}^{\dot{\rho}}=R_{\dot{\sigma} \alpha}^{\mp \gamma \dot{\delta}} R_{\gamma \dot{\delta}}^{ \pm \dot{\rho} \beta}
\end{aligned}
$$

\section{The quantum Lorentz group}

To have a correspondence between $S L(2, C)$ and Lorentz quantum groups, we must construct the quantum analogues of the Pauli matrices. Let us consider an element $X_{\alpha \dot{\beta}}$ as a tensor product of an undotted and dotted elements of right invariant basis of the bimodule $\mathcal{A}-\Gamma$ (bispinor). $X_{\alpha \dot{\beta}}$ can always be expanded on a system of four independent $2 \times 2$ matrices $\sigma_{\alpha \dot{\beta}}^{I}(I=0, . ., 3)$ as $X_{I} \sigma_{\alpha \dot{\beta}}^{I} . X_{\alpha \dot{\beta}}$ tranforms as

$$
\Delta_{L}\left(X_{\alpha \dot{\beta}}\right)=M_{\alpha}^{\sigma} M_{\dot{\beta}}^{\dot{\rho}} \otimes X_{\sigma \dot{\rho}} \quad, \quad \Delta_{R}\left(X_{\alpha \dot{\beta}}\right)=X_{\alpha \dot{\beta}} \otimes I
$$


Then, we have

\section{Proposition $(3,1)$ :}

a) There exist four $2 \times 2$ matrices $\bar{\sigma}_{ \pm}^{I \dot{\alpha} \beta}$ given by

$$
\bar{\sigma}_{ \pm}^{I \dot{\alpha} \beta}=\varepsilon^{\dot{\alpha} \dot{\lambda}} R_{\dot{\lambda} \nu}^{\mp \sigma \dot{\rho}} \varepsilon^{\nu \beta} \sigma_{\sigma \dot{\rho}}^{I}
$$

such that $X_{I} \bar{\sigma}_{ \pm}^{I \dot{\alpha} \beta}=X_{ \pm}^{\dot{\alpha} \beta}$ transforms under the left coaction as

$$
\Delta_{L}\left(X_{ \pm}^{\dot{\alpha} \beta}\right)=S^{-1}\left(M_{\dot{\sigma}}^{\dot{\alpha}}\right) S\left(M_{\rho}^{\beta}\right) \otimes X_{ \pm}^{\dot{\sigma} \rho} .
$$

b) $\bar{\sigma}_{ \pm}^{I \dot{\alpha} \beta}$ are hermitean iff ${\sigma_{\alpha \dot{\beta}}^{I}}$ are. In this case $X_{I}$ are real.

Proof.: a) Setting into (21) $M_{\alpha}{ }^{\sigma} M_{\dot{\beta}}^{\dot{\rho}}=R_{\alpha \dot{\beta}}^{ \pm \dot{\gamma} \delta} M_{\dot{\gamma}}^{\dot{\mu}} M_{\delta}{ }^{\nu} R_{\dot{\mu} \nu}^{\mp \sigma \dot{\rho}}$, obtained by multiplying from the right both sides of (18) by $R_{\dot{\rho} \beta}^{\mp \xi \dot{\tau}}$ and by using (19), we obtain

$$
\Delta_{L}\left(X_{\alpha \dot{\beta}}\right)=R_{\alpha \dot{\beta}}^{ \pm \dot{\gamma} \delta} M_{\dot{\gamma}}^{\dot{\mu}} M_{\delta}^{\nu} R_{\dot{\mu} \nu}^{\mp \sigma \dot{\rho}} \otimes X_{\sigma \dot{\rho}}
$$

Multiplying from the left both sides by $R_{\dot{\lambda} \nu}^{\mp \alpha \dot{\beta}}$ and using (20), we deduce

$$
\begin{gathered}
\Delta_{L}\left(X_{I} R_{\dot{\lambda} \nu}^{\mp \alpha \dot{\beta}} \sigma_{\alpha \dot{\beta}}^{I}\right)=M_{\dot{\lambda}}^{\dot{\mu}} M_{\nu}^{\tau} \otimes X_{I} R_{\dot{\mu} \tau}^{\mp \sigma \dot{\rho}} \sigma_{\sigma \dot{\rho}}^{I}= \\
\varepsilon_{\dot{\lambda} \dot{\gamma}} S^{-1}\left(M_{\dot{\delta}}^{\dot{\gamma}}\right) \varepsilon^{\dot{\delta} \dot{\mu}} \varepsilon^{\tau \xi} S\left(M_{\xi}{ }^{\kappa}\right) \varepsilon_{\kappa \nu} \otimes X_{I} R_{\dot{\mu} \tau}^{\mp \sigma \dot{\rho}} \sigma_{\sigma \dot{\rho}}^{I}
\end{gathered}
$$

yielding

$$
\Delta_{L}\left(X_{I} \varepsilon^{\dot{\alpha} \dot{\lambda}} R_{\dot{\lambda} \nu}^{\mp \sigma \dot{\rho}} \varepsilon^{\nu \beta} \sigma_{\sigma \dot{\rho}}^{I}\right)=S^{-1}\left(M_{\dot{\delta}}^{\dot{\alpha}}\right) S\left(M_{\gamma}^{\beta}\right) \otimes X_{I} \varepsilon^{\dot{\delta} \dot{\lambda}} R_{\dot{\lambda} \nu}^{\mp \sigma \dot{\rho}} \varepsilon^{\nu \gamma} \sigma_{\sigma \dot{\rho}}^{I}
$$

which can be written under the form $\Delta_{L}\left(X_{ \pm}^{\dot{\alpha} \beta}\right)=\Delta_{L}\left(X_{I} \bar{\sigma}_{ \pm}^{I \dot{\alpha} \beta}\right)=S^{-1}\left(M_{\dot{\delta}}{ }^{\dot{\alpha}}\right) S\left(M_{\gamma}^{\beta}\right) \otimes X_{ \pm}^{\dot{\delta} \gamma}$ with

$$
\bar{\sigma}_{ \pm}^{I \dot{\alpha} \beta}=\varepsilon^{\dot{\alpha} \dot{\lambda}} R_{\dot{\lambda} \nu}^{\mp \sigma \dot{\rho}} \varepsilon^{\nu \beta} \sigma_{\sigma \dot{\rho}}^{I}
$$

from which we obtain $\varepsilon_{\dot{\lambda} \dot{\alpha}} \bar{\sigma}_{ \pm}^{I \dot{\alpha} \beta} \varepsilon_{\beta \nu}=R_{\dot{\lambda} \nu}^{\mp \sigma \dot{\rho}} \sigma_{\sigma \dot{\rho}}^{I}$. Multiplying from the right both sides by $R_{\gamma \dot{\tau}}^{ \pm \dot{\lambda} \nu}$ and using (19), we get

$$
\sigma_{\alpha \dot{\beta}}^{I}=\varepsilon_{\dot{\lambda} \dot{\gamma}} R_{\alpha \dot{\beta}}^{ \pm \dot{\lambda} \nu} \varepsilon_{\mu \nu} \bar{\sigma}_{ \pm}^{I \dot{\gamma} \mu}
$$

b) Under the conditions (16) on the spinor metric we have $\left(R_{\sigma \nu}^{ \pm \alpha \rho}\right)^{\star}=R_{\alpha \rho}^{ \pm \sigma \nu}$, for $a$ real, implying

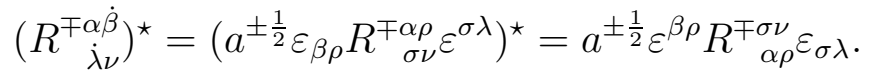


In the other hand, by using (11) and (15), we obtain

$$
\begin{array}{r}
a^{ \pm \frac{1}{2}} \varepsilon^{\beta \rho} R_{\alpha \rho}^{\mp \sigma \nu} \varepsilon_{\sigma \lambda}=f_{\mp \alpha}^{\nu}\left(\varepsilon^{\beta \rho} M_{\rho}{ }^{\sigma} \varepsilon_{\sigma \lambda}\right)=f_{\mp \alpha}^{\nu}\left(S^{-1}\left(M_{\lambda}{ }^{\beta}\right)\right)=\tilde{f}_{\mp \alpha}^{\nu}\left(M_{\lambda}{ }^{\beta}\right)= \\
\varepsilon_{\alpha \sigma} f_{\mp \delta}{ }^{\sigma}\left(M_{\lambda}{ }^{\beta}\right) \varepsilon^{\delta \nu}=a^{ \pm \frac{1}{2}} \varepsilon_{\alpha \sigma} R_{\delta \lambda}^{\mp \beta \sigma} \varepsilon^{\delta \nu}=R_{\dot{\nu} \lambda}^{\mp \beta} \dot{\alpha}
\end{array}
$$

yielding $\left(\bar{\sigma}_{ \pm}^{I \dot{\alpha} \beta}\right)^{\star}=\varepsilon^{\dot{\beta} \dot{\nu}} R_{\dot{\nu} \lambda}^{\mp \rho \dot{\sigma}} \varepsilon^{\lambda \alpha}\left(\sigma_{\sigma \dot{\rho}}^{I}\right)^{\star}$. Therefore, if $\left(\sigma_{\sigma \dot{\rho}}^{I}\right)^{\star}=\sigma_{\rho \dot{\sigma}}^{I}$ then

$$
\left(\bar{\sigma}_{ \pm}^{I \dot{\alpha} \beta}\right)^{\star}=\varepsilon^{\dot{\beta} \dot{\nu}} R_{\dot{\nu} \lambda}^{\mp \rho \dot{\sigma}} \varepsilon^{\lambda \alpha} \sigma_{\rho \dot{\sigma}}^{I}=\bar{\sigma}_{ \pm}^{I \dot{\beta} \alpha}
$$

The same procedure can be applyied to (23) to show the converse. It is now easy to see by applying the $\star$ involution on both sides of $(21)$ that $\left(X_{\alpha \dot{\beta}}\right)^{\star}=X_{\beta \dot{\alpha}} \operatorname{implying} X_{I} \sigma_{\beta \dot{\alpha}}^{I}=\left(X_{I} \sigma_{\alpha \dot{\beta}}^{I}\right)^{\star}=$ $\left(X_{I}\right)^{\star} \sigma_{\beta \dot{\alpha}}^{I}$ which shows that $X_{I}$ are real. Q.E.D.

To define a quantum metric of the space $\mathcal{M}$ spanned by $X_{I}$, we have to define an adequate trace [10-11] over the spinorial indices which makes invariant this metric under quantum $S L(2, C)$ group.

\section{Proposition $(3,2)$ :}

$\mathcal{M}$ is endowed with a metric $G^{I J}$ given by

$$
G_{ \pm}^{I J}=\frac{1}{Q} \operatorname{Tr}\left(\sigma^{I} \bar{\sigma}_{ \pm}^{J}\right)=\frac{1}{Q} \varepsilon^{\alpha \nu} \sigma_{\alpha \dot{\beta}}^{I} \bar{\sigma}_{ \pm}^{J \dot{\beta} \gamma} \varepsilon_{\gamma \nu}=\frac{1}{Q} \operatorname{Tr}\left(\bar{\sigma}_{ \pm}^{I} \sigma^{J}\right)=\frac{1}{Q} \varepsilon_{\dot{\nu} \dot{\gamma}} \bar{\sigma}_{ \pm}^{I \dot{\gamma} \alpha} \sigma_{\alpha \dot{\beta}}^{J} \varepsilon^{\dot{\nu} \dot{\beta}}
$$

such that

a) $G_{ \pm}^{I J} X_{I} X_{J}$ are invariant under quantum $S L(2, C)$ group.

b) $G_{ \pm}^{I J}$ are hermitean if the matrices $\sigma_{\alpha \dot{\beta}}^{I}$ are.

Proof: a) To show the invariance of $G$ under the quantum $S L(2, C)$ group, we consider the norm of $X$ as $G_{ \pm}^{I J} X_{I} X_{J}=\frac{1}{Q} \varepsilon^{\alpha \nu} X_{\alpha \dot{\beta}} X_{ \pm}^{\dot{\beta} \gamma} \varepsilon_{\gamma \nu}$ which transforms under $S L(2, C)$ as

$$
\begin{array}{r}
\Delta_{L}\left(G_{ \pm}^{I J} X_{I} X_{J}\right)=\frac{1}{Q} \varepsilon^{\alpha \nu} M_{\alpha}{ }^{\sigma} M_{\dot{\beta}}^{\dot{\rho}} S^{-1}\left(M_{\dot{\delta}}^{\dot{\beta}}\right) S\left(M_{\lambda}^{\gamma}\right) \varepsilon_{\gamma \nu} \otimes X_{\sigma \dot{\rho}} X_{ \pm}^{\dot{\delta} \lambda}= \\
\frac{1}{Q} \varepsilon^{\alpha \nu} M_{\alpha}{ }^{\sigma} S\left(M_{\lambda}^{\gamma}\right) \varepsilon_{\gamma \nu} \otimes X_{\sigma \dot{\delta}} X_{ \pm}^{\dot{\delta} \lambda}=\frac{1}{Q} \varepsilon^{\alpha \nu} M_{\alpha}^{\sigma} \varepsilon_{\lambda \rho} M_{\mu}{ }^{\rho} \varepsilon^{\mu \gamma} \varepsilon_{\gamma \nu} \otimes X_{\sigma \dot{\delta}} X_{ \pm}^{\dot{\delta} \lambda}= \\
I \otimes \frac{1}{Q} \varepsilon^{\sigma \rho} X_{\sigma \dot{\delta}} X_{ \pm}^{\dot{\delta} \lambda} \varepsilon_{\lambda \rho}=I \otimes \frac{1}{Q} \varepsilon^{\sigma \rho} \sigma_{\sigma \dot{\delta}}^{I} \bar{\sigma}_{ \pm}^{J \dot{\delta} \lambda} \varepsilon_{\lambda \rho} X_{I} X_{J}=I \otimes G_{ \pm}^{I J} X_{I} X_{J}
\end{array}
$$

The same computation may be applyed to show that $\varepsilon_{\dot{\nu} \dot{\gamma}} X_{ \pm}^{\dot{\gamma} \alpha} X_{\alpha \dot{\beta}} \varepsilon^{\dot{\nu} \dot{\beta}}$ is invariant under quantum $S L(2, C)$ group.

Now, using (22) and the form of the $\mathrm{R}$ matrices, we obtain

$$
G_{ \pm}^{I J}=\frac{1}{Q} \varepsilon^{\alpha \xi} \sigma_{\alpha \dot{\beta}}^{I} \varepsilon^{\dot{\beta} \dot{\lambda}} R_{\dot{\lambda} \nu}^{\mp \sigma \dot{\rho}} \varepsilon^{\nu \gamma} \sigma_{\sigma \dot{\rho}}^{J} \varepsilon_{\gamma \xi}=\frac{1}{Q} a^{ \pm \frac{1}{2}} \varepsilon^{\alpha \xi} \sigma_{\alpha \dot{\beta}}^{I} \varepsilon^{\dot{\beta} \dot{\lambda}} \varepsilon_{\rho \delta} R_{\mu \nu}^{\mp \sigma \delta} \varepsilon^{\mu \lambda} \varepsilon^{\nu \gamma} \sigma_{\sigma \dot{\rho}}^{J} \varepsilon_{\gamma \xi}=
$$




$$
\begin{array}{r}
\frac{1}{Q} a^{ \pm \frac{1}{2}} \varepsilon^{\alpha \xi} \varepsilon^{\dot{\beta} \dot{\lambda}_{\rho}} \varepsilon_{\rho \delta}\left(\delta_{\mu}^{\sigma} \delta_{\xi}^{\delta}+a^{\mp 1} \varepsilon^{\sigma \delta} \varepsilon_{\mu \xi}\right) \varepsilon^{\mu \lambda} \sigma_{\alpha \dot{\beta}}^{I} \sigma_{\sigma \dot{\rho}}^{J}= \\
-\frac{1}{Q} a^{ \pm \frac{1}{2}} \varepsilon^{\alpha \xi} \varepsilon^{\dot{\beta} \lambda} \varepsilon_{\rho \delta}\left(\delta_{\mu}^{\sigma} \delta_{\xi}^{\delta}+a^{\mp 1} \varepsilon^{\sigma \delta} \varepsilon_{\mu \xi}\right) \varepsilon_{d o t \lambda \dot{\mu}} \sigma_{\alpha \dot{\beta}}^{I} \sigma_{\sigma \dot{\rho}}^{J}= \\
-\frac{1}{Q} a^{ \pm \frac{1}{2}} \varepsilon^{\alpha \xi} \varepsilon_{\rho \delta}\left(\delta_{\mu}^{\sigma} \delta_{\xi}^{\delta}+a^{\mp 1} \varepsilon^{\sigma \delta} \varepsilon_{\mu \xi}\right) \sigma_{\alpha \dot{\mu}}^{I} \sigma_{\sigma \dot{\rho}}^{J}= \\
\frac{1}{Q}\left(a^{ \pm \frac{1}{2}} \sigma^{I \xi}{ }_{\dot{\mu}} \sigma_{\mu}^{J \dot{\xi}}-a^{\mp \frac{1}{2}} \sigma^{I \xi \dot{\xi}} \sigma^{J \delta \dot{\delta}}\right)
\end{array}
$$

where we have used (16) in the third and fifth line and the $\sigma^{I}$ indices are raised and lowered as for the basis of the bicovariant $\mathcal{A}-\Gamma$ bimodule $\left(\sigma_{\dot{\beta}}^{I \alpha}=\sigma_{\rho \dot{\beta}}^{I} \varepsilon^{\rho \alpha}, \sigma_{\alpha}^{I \dot{\beta}}=\varepsilon^{\dot{\beta} \dot{\rho}} \sigma_{\alpha \dot{\rho}}^{I}\right.$ etc,..). From a similar computation we can show that $\operatorname{Tr}\left(\bar{\sigma}_{ \pm}^{I} \sigma^{J}\right)$ gives the same form (25) for $G_{ \pm}{ }^{I J}$.

b) if $\sigma_{\alpha \dot{\beta}}^{I}$ are hermitean, we have from (16) and the proposition(3,1)

$$
\left(G_{ \pm}^{I J}\right)^{\star}=\frac{1}{Q}\left(\varepsilon^{\alpha \nu} \sigma_{\alpha \dot{\beta}}^{I} \bar{\sigma}_{ \pm}^{J \dot{\beta} \gamma} \varepsilon_{\gamma \nu}\right)^{\star}=\frac{1}{Q} \varepsilon_{\dot{\nu} \dot{\gamma}} \bar{\sigma}_{ \pm}^{J \dot{\gamma} \beta} \sigma_{\beta \dot{\alpha}}^{I} \varepsilon^{\dot{\nu} \dot{\alpha}}=G_{ \pm}^{J I} .
$$

which shows that the metric $G_{ \pm}^{I J}$ is hermitean. Q.E.D.

Note that if $\sigma_{\alpha \dot{\beta}}^{I}$ are hermitean, then as well as $X_{I}$ as its norm $G_{ \pm}^{I J} X_{I} X_{J}$ are real.

Now, we can give an explicit example where we take $\sigma^{I}$ the usual four matrices which are the $2 \times 2$ identity matrix $\sigma_{\alpha \dot{\beta}}^{0}$ and the three Pauli matrices $\sigma_{\alpha \dot{\beta}}^{i}(i=1,2,3)$ as:

$$
\sigma_{\alpha \dot{\beta}}^{0}=\left(\begin{array}{cc}
1 & 0 \\
0 & 1
\end{array}\right), \quad \sigma_{\alpha \dot{\beta}}^{1}=\left(\begin{array}{cc}
0 & 1 \\
1 & 0
\end{array}\right), \quad \sigma_{\alpha \dot{\beta}}^{2}=\left(\begin{array}{cc}
0 & -i \\
i & 0
\end{array}\right), \quad \sigma_{\alpha \dot{\beta}}^{3}=\left(\begin{array}{cc}
1 & 0 \\
0 & -1
\end{array}\right) .
$$

For the spinorial metric satisfying (16), we may take the most general form as

$$
\varepsilon_{\alpha \beta}=d^{-\frac{1}{2}}\left(\begin{array}{cc}
i r & -q^{-\frac{1}{2}} \\
q^{\frac{1}{2}} & i r
\end{array}\right), \quad \varepsilon^{\alpha \beta}=d^{-\frac{1}{2}}\left(\begin{array}{cc}
i r & q^{-\frac{1}{2}} \\
-q^{\frac{1}{2}} & i r
\end{array}\right)
$$

and

$$
\varepsilon_{\dot{\alpha} \dot{\beta}}=d^{-\frac{1}{2}}\left(\begin{array}{cc}
-i r & q^{\frac{1}{2}} \\
-q^{-\frac{1}{2}} & -i r
\end{array}\right), \quad \varepsilon^{\dot{\alpha} \dot{\beta}}=d^{-\frac{1}{2}}\left(\begin{array}{cc}
-i r & -q^{\frac{1}{2}} \\
q^{-\frac{1}{2}} & -i r
\end{array}\right)
$$

where $q$ and $r \neq \pm 1$ are real, $d=-r^{2}+1$ and $Q=a+a^{-1}=d^{-1}\left(2 r+q+q^{-1}\right)=-\varepsilon_{\alpha \beta} \varepsilon^{\alpha \beta}$. With this choice, the computer MAPLE program gives metrics $G_{ \pm}^{I J}$ of the form

$$
\left(\begin{array}{clcr}
-A_{(1)} & 0 & 2 A_{(1)} r d^{-1}\left(\frac{Q_{\left(\frac{1}{2}\right)}}{Q}\right) & A_{(1)} d^{-1} Q_{(-)} \\
0 & A_{(2)} & -i A_{(2)} d^{-1} Q_{(-)} & 2 i A_{(2)} r d^{-1}\left(\frac{Q_{\left(\frac{1}{2}\right)}}{Q}\right) \\
2 A_{(1)} r d^{-1}\left(\frac{Q_{\left(\frac{1}{2}\right)}}{Q}\right) & i A_{(2)} d^{-1} Q_{(-)} & A_{(2)}-4 A_{(3)} r^{2} d^{-2}\left(\frac{\left(Q_{\left(\frac{1}{2}\right)}\right)^{2}}{Q}\right) & -2 A_{(2)} r d^{-2} Q_{(-)} Q_{\left(\frac{1}{2}\right)} \\
A_{(1)} d^{-1} Q(-) & -2 i A_{(2)} r d^{-1}\left(\frac{Q_{\left(\frac{1}{2}\right)}}{Q}\right) & -2 A_{(2)} r d^{-2} Q_{(-)} Q_{\left(\frac{1}{2}\right)} & A_{(2)}-A_{(3)} d^{-2}\left(Q_{(-)}\right)^{2} Q
\end{array}\right)
$$


where $A_{(1)}=a^{\mp \frac{3}{2}}, A_{(2)}=a^{ \pm \frac{1}{2}}, A_{(3)}=a^{\mp \frac{1}{2}}, Q_{\left(\frac{1}{2}\right)}=q^{\frac{1}{2}}+q^{-\frac{1}{2}}$ and $Q_{(-)}=\frac{q-q^{-1}}{Q}$. The inverse $G_{ \pm I J}$ is given by

$$
\left(\begin{array}{clrr}
-A_{(4)} \frac{Q^{2}}{4}+\frac{d^{-1}}{4} A_{(2)}\left(Q_{\left(\frac{1}{2}\right)}\right)^{2}(Q-2) Q & 0 & \frac{r d^{-1}}{2} A_{(3)} Q_{\left(\frac{1}{2}\right)} Q & \frac{d^{-1}}{4} A_{(3)} Q_{(-)} Q^{2} \\
0 & \frac{Q^{2}}{4} A_{(3)} & i \frac{d^{-1}}{4} A_{(3)} Q_{(-)} Q^{2} & -i \frac{r d^{-1}}{2} A_{(3)} Q_{\left(\frac{1}{2}\right)} Q \\
\frac{r d^{-1}}{2} A_{(3)} Q_{\left(\frac{1}{2}\right)} Q & -i \frac{d^{-1}}{4} A_{(3)} Q_{(-)} Q^{2} & \frac{Q^{2}}{4} A_{(3)} & 0 \\
\frac{d^{-1}}{4} A_{(3)} Q_{(-)} Q^{2} & i \frac{r d^{-1}}{2} A_{(3)} Q_{\left(\frac{1}{2}\right)} Q & 0 & \frac{Q^{2}}{4} A_{(3)}
\end{array}\right)
$$

where $A_{(4)}=a^{ \pm \frac{3}{2}}$. In the classical limit $r=0$ and $q=1$, these metrics reduce to the classical Minkowsky metric with signature $(-,+,+,+)$.

From the computer MAPLE program, we obtain the completeness relations as

$$
\sigma_{\alpha \dot{\beta}}^{I} \bar{\sigma}_{I}^{\dot{\rho} \sigma}=Q \delta_{\alpha}^{\sigma} \varepsilon_{\dot{\beta} \dot{\delta}} \varepsilon^{\dot{\rho} \dot{\delta}} \quad, \quad \sigma_{ \pm I \alpha \dot{\beta}} \bar{\sigma}_{ \pm}^{I \dot{\rho} \sigma}=Q \delta_{\dot{\beta}}^{\dot{\rho}} \varepsilon_{\delta \alpha} \varepsilon^{\delta \sigma}
$$

or

$$
\sigma_{\alpha}^{I}{ }^{\dot{\beta}} \bar{\sigma}_{I \dot{\rho}}^{\sigma}=Q \delta_{\alpha}^{\sigma} \delta_{\dot{\rho}}^{\dot{\beta}} \quad, \quad \sigma_{ \pm I \dot{\beta}}^{\alpha} \bar{\sigma}_{ \pm \sigma}^{I \dot{\rho}}=Q \delta_{\sigma}^{\alpha} \delta_{\dot{\beta}}^{\dot{\rho}}
$$

where $\sigma_{ \pm I \alpha \dot{\beta}}=G_{ \pm I J} \sigma_{\alpha \dot{\beta}}^{J}$. Note that from the computer MAPLE program we can check that $\bar{\sigma}_{J}^{\dot{\alpha} \beta}=G_{+I J} \bar{\sigma}_{+}^{J \dot{\alpha} \beta}=G_{-I J} \bar{\sigma}_{-}^{J \dot{\alpha} \beta}=G_{ \pm I J} \bar{\sigma}_{ \pm}^{J \dot{\alpha} \beta}$ and $\bar{\sigma}_{+}^{J \dot{\alpha} \beta} G_{+J I}=\bar{\sigma}_{-}^{J \dot{\alpha} \beta} G_{-J I}$.

$\operatorname{Remark}(3,1)$ :

- The metric $G_{ \pm I J}$ can be written as $G_{ \pm I J}=G_{ \pm I L} G_{ \pm J K} G_{ \pm}^{K L}=G_{ \pm I L} G_{ \pm J K} \frac{1}{Q} \operatorname{Tr}\left(\sigma^{K} \bar{\sigma}_{ \pm}^{L}\right)=$ $\frac{1}{Q} \operatorname{Tr}\left(\sigma_{ \pm J} \bar{\sigma}_{I}\right)=G_{ \pm I L} G_{ \pm J K} \frac{1}{Q} \operatorname{Tr}\left(\bar{\sigma}_{ \pm}^{K} \sigma^{L}\right)=\frac{1}{Q} \operatorname{Tr}\left(\bar{\sigma}_{J} \sigma_{ \pm I}\right)$. With a way quite analogous that was used to derive (25), we may show that

$$
G_{ \pm I J}=\frac{1}{Q}\left(a^{\mp \frac{1}{2}} \bar{\sigma}_{I \delta}^{\dot{\beta}} \bar{\sigma}_{J \dot{\delta}}^{\beta}-a^{ \pm \frac{1}{2}} \bar{\sigma}_{I \dot{\delta} \delta} \bar{\sigma}_{J \dot{\beta} \beta}\right)
$$

- The completeness relations (27) may be used to convert a vector to a bispinor and vice versa

$$
X_{\alpha \dot{\beta}}=X_{I} \sigma_{\alpha \dot{\beta}}^{I} \Leftrightarrow X_{I}=\frac{1}{Q} \varepsilon^{\alpha \nu} X_{\alpha \dot{\beta}} \bar{\sigma}_{ \pm}^{J \dot{\beta} \delta} \varepsilon_{\delta \nu} G_{ \pm J I} \quad \text { or } \quad X_{I}=\frac{1}{Q} \varepsilon_{\dot{\nu} \dot{\beta}} \bar{\sigma}_{I}^{\dot{\beta} \alpha} X_{\alpha \dot{\delta}} \varepsilon^{\dot{\nu} \dot{\delta}}
$$

We are now ready to show how two copies of undotted and dotted generators of $\mathcal{A}$ may be combined to form generators $\Lambda_{L}{ }^{K}$ of an unital algebra $\mathcal{L}$ corresponding to the quantum Lorentz group.

Theorem $(3,1)$ :

The generators $\Lambda_{L}^{K}(L, K=0,1,2,3)$ of the Quantum Lorentz group are given by

$$
\Lambda_{L}^{K}=\frac{1}{Q} \varepsilon_{\dot{\gamma} \dot{\delta}} \bar{\sigma}_{L}^{\dot{\delta} \alpha} M_{\alpha}{ }^{\sigma} \sigma_{\sigma \dot{\rho}}^{K} M_{\dot{\beta}}^{\dot{\rho}} \varepsilon^{\dot{\gamma} \dot{\beta}}=\frac{1}{Q} \bar{\sigma}_{L \dot{\gamma}}^{\alpha} M_{\alpha}^{\sigma} \sigma_{\sigma}^{K}{ }_{\sigma}^{\dot{\rho}} S^{-1}\left(M_{\dot{\rho}}^{\dot{\gamma}}\right)
$$


or

$$
\Lambda_{L}^{K}=\frac{1}{Q} \varepsilon^{\alpha \delta} M_{\alpha}^{\sigma} \sigma_{\sigma \dot{\rho}}^{K} M_{\dot{\beta}}^{\dot{\rho}} \bar{\sigma}_{ \pm}^{N \dot{\beta} \gamma} \varepsilon_{\gamma \delta} G_{ \pm N L}
$$

They are real and satisfy the axiomatic structure of Hopf algebras with the relations

$$
G_{ \pm N M} \Lambda_{L}^{N} \Lambda_{K}^{M}=G_{ \pm L K} I_{\mathcal{L}} \text { and } G_{ \pm}^{L K} \Lambda_{L}^{N} \Lambda_{K}^{M}=G_{ \pm}^{N M} I_{\mathcal{L}}
$$

where $I_{\mathcal{L}}=I_{\mathcal{A}}$ is the unity of $\mathcal{L} \subset \mathcal{A}$.

Proof: Multiplying from the left both sides of (21) by $\bar{\sigma}^{N \dot{\delta} \alpha}$ and making the trace over dotted indices, we get

$$
\Delta_{L} X_{L} \operatorname{Tr}\left(\bar{\sigma}_{ \pm}^{N \dot{\delta} \alpha} \sigma_{\alpha \dot{\beta}}^{L}\right)=\Delta_{L} X_{L} Q G_{ \pm}^{N L}=\varepsilon_{\dot{\gamma} \dot{\delta}} \bar{\sigma}_{ \pm}^{N \dot{\delta} \alpha} M_{\alpha}^{\sigma} \sigma_{\sigma \dot{\rho}}^{K} M_{\dot{\beta}}^{\dot{\rho}} \varepsilon^{\dot{\gamma} \dot{\beta}} \otimes X_{K}
$$

yielding

$$
\Delta_{L} X_{L}=\frac{1}{Q} \varepsilon_{\dot{\gamma} \dot{\delta}} \bar{\sigma}_{L}^{\dot{\delta} \alpha} M_{\alpha}{ }^{\sigma} \sigma_{\sigma \dot{\rho}}^{K} M_{\dot{\beta}}^{\dot{\rho}} \varepsilon^{\dot{\gamma} \dot{\beta}} \otimes X_{K}=\Lambda_{L}{ }^{K} \otimes X_{K}
$$

We can also multiply from the right both sides of (21) by $\bar{\sigma}_{ \pm}^{N \dot{\beta} \gamma}$ and take trace over undotted indices to have (30).

To show that (29) is equal to (30), we note that

$$
\begin{array}{r}
\varepsilon^{\alpha \delta} \bar{\sigma}_{ \pm}^{N \dot{\beta} \gamma} \varepsilon_{\gamma \delta} G_{ \pm N L}=\frac{1}{Q} \varepsilon^{\alpha \delta} \bar{\sigma}_{ \pm}^{N \dot{\beta} \gamma} \varepsilon_{\gamma \delta} \varepsilon_{\dot{\nu} \dot{\mu}} \bar{\sigma}_{L}^{\dot{\mu} \rho} \sigma_{ \pm N \rho \dot{\tau}} \varepsilon^{\dot{\nu} \dot{\tau}}= \\
\varepsilon^{\alpha \delta} \delta_{\dot{\tau}}^{\dot{\beta}} \varepsilon_{\sigma \rho} \varepsilon^{\sigma \gamma} \varepsilon_{\gamma \delta} \varepsilon_{\dot{\nu} \dot{\mu}} \bar{\sigma}_{L}^{\dot{\mu} \rho} \varepsilon^{\dot{\nu} \dot{\tau}}=\varepsilon_{\dot{\nu} \dot{\mu}} \bar{\sigma}_{L}^{\dot{\mu} \alpha} \varepsilon^{\dot{\nu} \dot{\beta}}
\end{array}
$$

where we have used $G_{ \pm N L}=\operatorname{Tr}\left(\bar{\sigma}_{L} \sigma_{ \pm N}\right)$ and the completeness relation (27). Substituting this equality in (30), we retrieve (29).

The reality of the generators is obtained by noticing that $\left(\bar{\sigma}_{ \pm}^{N \dot{\beta} \gamma} G_{ \pm N L}\right)^{\star}=G_{ \pm L N} \bar{\sigma}_{ \pm}^{N \dot{\gamma} \beta}=\bar{\sigma}_{L}^{\dot{\gamma} \beta}$ from which, we get

$$
\left(\Lambda_{L}^{K}\right)^{\star}=\left(\frac{1}{Q} \varepsilon^{\alpha \delta} M_{\alpha}^{\sigma} \sigma_{\sigma \dot{\rho}}^{K} M_{\dot{\beta}}^{\dot{\rho}} \bar{\sigma}_{ \pm}^{N \dot{\beta} \gamma} \varepsilon_{\gamma \delta} G_{ \pm N L}\right)^{\star}=\frac{1}{Q} \varepsilon_{\dot{\delta} \dot{\gamma}} \bar{\sigma}_{L}^{\dot{\gamma} \beta} M_{\beta}{ }^{\rho} \sigma_{\rho \dot{\sigma}}^{K} M_{\dot{\alpha}}^{\dot{\sigma}} \varepsilon^{\dot{\delta} \dot{\alpha}}=\Lambda_{L}^{K}
$$

due to the fact that $\sigma_{\sigma \dot{\rho}}^{I}$ are hermitean. The Hopf structure of the algebra generated by $\Lambda_{L}{ }^{K}$ is given by:

a) Acting the coaction on both sides of (29), we obtain

$$
\Delta\left(\Lambda_{L}^{K}\right)=\frac{1}{Q} \bar{\sigma}_{L \dot{\gamma}}^{\alpha} M_{\alpha}^{\delta} S^{-1}\left(M_{\dot{\nu}}^{\dot{\gamma}}\right) \otimes M_{\lambda}^{\sigma} \sigma_{\sigma}^{K} \dot{\rho} S^{-1}\left(M_{\dot{\rho}}^{\dot{\mu}}\right) \delta_{\delta}^{\lambda} \delta_{\dot{\mu}}^{\dot{\nu}}
$$

From the completeness relation (27), we deduce

$$
\Delta\left(\Lambda_{L}{ }^{K}\right)=\frac{1}{Q} \bar{\sigma}_{L \dot{\gamma}}^{\alpha} M_{\alpha}{ }^{\delta} \sigma^{I}{ }_{\delta}^{\dot{\nu}} S^{-1}\left(M_{\dot{\nu}}^{\dot{\gamma}}\right) \otimes \frac{1}{Q} \bar{\sigma}_{I \dot{\mu}}{ }^{\delta} M_{\lambda}{ }^{\sigma} \sigma_{\sigma}^{K}{ }^{\dot{\rho}} S^{-1}\left(M_{\dot{\rho}}^{\dot{\mu}}\right)=\Lambda_{L}^{I} \otimes \Lambda_{I}^{K} .
$$


b)The counity acts as

$$
\begin{array}{r}
\varepsilon\left(\Lambda_{L}{ }^{K}\right)=\frac{1}{Q} \bar{\sigma}_{L \dot{\gamma}}{ }^{\alpha} \varepsilon\left(M_{\alpha}{ }^{\sigma}\right) \sigma_{\sigma}^{K}{ }_{\sigma}^{\dot{\rho}} \varepsilon\left(S^{-1}\left(M_{\dot{\rho}}^{\dot{\gamma}}\right)\right)=\frac{1}{Q} \bar{\sigma}_{L \dot{\gamma}}{ }^{\alpha} \delta_{\alpha}{ }^{\sigma} \sigma_{\sigma}^{K}{ }_{\sigma}^{\dot{\rho}} \dot{\rho}_{\dot{\rho}}^{\dot{\gamma}}= \\
\frac{1}{Q} \bar{\sigma}_{L \dot{\gamma}}{ }^{\alpha} \sigma_{\alpha}^{K}{ }_{\alpha}^{\dot{\gamma}}=\frac{1}{Q} G_{ \pm L N} \operatorname{Tr}\left(\bar{\sigma}_{ \pm}^{N} \sigma^{K}\right)=G_{ \pm L N} G_{ \pm}^{N K}=\delta_{L}^{K} .
\end{array}
$$

c) Finally, applying the antipode on both sides of (29), we get

$$
S\left(\Lambda_{L}^{K}\right)=\frac{1}{Q} \varepsilon^{\dot{\gamma} \dot{\beta}} S\left(M_{\dot{\beta}}^{\dot{\rho}}\right) \sigma_{\sigma \dot{\rho}}^{K} S\left(M_{\alpha}{ }^{\sigma}\right) \bar{\sigma}_{L}^{\dot{\delta} \alpha} \varepsilon_{\dot{\gamma} \dot{\delta}}=\frac{1}{Q} M_{\dot{\nu}}^{\dot{\gamma}} \varepsilon^{\dot{\nu} \dot{\rho}} \sigma_{\sigma \dot{\rho}}^{K} \varepsilon_{\alpha \lambda} M_{\mu}{ }^{\lambda} \varepsilon^{\mu \sigma} \bar{\sigma}_{L}^{\dot{\delta} \alpha} \varepsilon_{\dot{\gamma} \dot{\delta}} .
$$

It now follows from $M_{\dot{\nu}}^{\dot{\gamma}} M_{\mu}^{\lambda}=R_{\dot{\nu} \mu}^{\mp \tau \dot{\xi}} M_{\tau}^{\delta} M_{\dot{\xi}}^{\dot{\rho}} R_{\delta \dot{\rho}}^{ \pm \dot{\gamma} \lambda}$, obtained by multiplying from the right both sides of (18) by $R_{\dot{\tau} \nu}^{\mp \alpha \dot{\sigma}}$ and by using (19), that

$$
\begin{aligned}
& S\left(\Lambda_{L}^{K}\right)=\frac{1}{Q} R_{\dot{\nu} \mu}^{\mp \tau \dot{\xi}} M_{\tau}^{\delta} M_{\dot{\xi}}^{\dot{\beta}} R_{\delta \dot{\beta}}^{ \pm \dot{\gamma} \lambda} \varepsilon^{\dot{\nu} \dot{\rho}} \sigma_{\sigma \dot{\rho}}^{K} \varepsilon_{\alpha \lambda} \varepsilon^{\mu \sigma} \bar{\sigma}_{L}^{\dot{\delta} \alpha} \varepsilon_{\dot{\gamma} \dot{\delta}}= \\
& \frac{1}{Q} R_{\dot{\nu} \mu}^{\mp \tau \dot{\xi}} M_{\tau}^{\delta} M_{\dot{\xi}}^{\dot{\beta}} \varepsilon^{\dot{\nu} \dot{\rho}} \sigma_{\sigma \dot{\rho}}^{K} \varepsilon^{\mu \sigma} \varepsilon_{\dot{\gamma} \dot{\delta}} R_{\delta \dot{\beta}}^{ \pm \dot{\gamma} \lambda} \varepsilon_{\alpha \lambda} \bar{\sigma}_{L}^{\dot{\delta} \alpha}= \\
& \frac{1}{Q} \varepsilon^{\mu \sigma} \sigma_{\sigma \dot{\rho}}^{K} \varepsilon^{\dot{\nu} \dot{\rho}} R_{\dot{\nu} \mu}^{\mp \dot{\xi}} M_{\tau}^{\delta} M_{\dot{\xi}}^{\dot{\beta}} \sigma_{ \pm L \delta \dot{\beta}}= \\
& \frac{1}{Q} R_{\dot{\nu} \mu}^{\mp \tau \dot{\xi}} \varepsilon^{\mu \sigma} \sigma_{\sigma}^{K} \dot{\nu} M_{\tau}^{\delta} M_{\dot{\xi}}^{\dot{\beta}} \sigma_{ \pm L \delta \dot{\beta}}
\end{aligned}
$$

where we have used (23) and $G_{ \pm L I} \sigma_{\alpha \dot{\beta}}^{I}=\sigma_{ \pm L \alpha \dot{\beta}}$ to pass from the second line to the third. In the other hand, we have

$$
\bar{\sigma}_{N}^{\dot{\alpha} \beta} G_{ \pm}^{N K}=\frac{1}{Q} \bar{\sigma}_{N}^{\dot{\alpha} \beta} \varepsilon_{\dot{\nu} \dot{\gamma}} \bar{\sigma}_{ \pm}^{N \dot{\gamma} \delta} \sigma_{\delta \dot{\tau}}^{K} \varepsilon^{\dot{\nu} \dot{\tau}}=\frac{1}{Q} \bar{\sigma}_{N}^{\dot{\alpha} \beta} \varepsilon_{\dot{\nu} \dot{\gamma}} \varepsilon^{\dot{\gamma} \dot{\lambda}} R_{\dot{\lambda} \sigma}^{\mp \tau \dot{\rho}} \varepsilon^{\sigma \delta} \sigma_{\tau \dot{\rho}}^{N} \sigma_{\delta \dot{\tau}}^{K} \varepsilon^{\dot{\nu} \dot{\tau}}
$$

where we have used (24) and (22). Using the completeness relation (26), we obtain

$$
\bar{\sigma}_{N}^{\dot{\alpha} \beta} G_{ \pm}^{N K}=\delta_{\tau}^{\beta} \varepsilon_{\dot{\rho} \dot{\mu}} \varepsilon^{\dot{\alpha} \dot{\mu}} R_{\dot{\nu} \sigma}^{\mp \tau \dot{\rho}} \varepsilon^{\sigma \delta} \sigma_{\delta \dot{\tau}}^{K} \varepsilon^{\dot{\nu} \dot{\tau}}
$$

or

$$
\bar{\sigma}_{N \dot{\alpha}}^{\beta} G_{ \pm}^{N K}=\varepsilon_{\dot{\rho} \dot{\alpha}} R_{\dot{\nu} \sigma}^{\mp \beta \dot{\rho}} \varepsilon^{\sigma \delta} \sigma_{\delta}^{K} \dot{\nu} .
$$

Substituting this equality in (35), we get

$$
\begin{array}{r}
S\left(\Lambda_{L}^{K}\right)=\frac{1}{Q} \bar{\sigma}_{N \dot{\alpha}}{ }^{\tau} M_{\tau}{ }^{\delta} \sigma_{L \delta \dot{\beta}} M_{\dot{\rho}} \dot{\dot{\beta}} \varepsilon^{\dot{\alpha} \dot{\rho}} G_{ \pm}{ }^{N K}= \\
\frac{1}{Q} G_{ \pm L M}\left(\bar{\sigma}_{N \dot{\alpha}}{ }^{\tau} M_{\tau}{ }^{\delta} \sigma_{\delta \dot{\beta}}^{M} M_{\dot{\rho}}^{\dot{\beta}} \varepsilon^{\dot{\alpha} \dot{\rho}}\right) G_{ \pm}^{N K}=G_{ \pm L M} \Lambda_{N}^{M} G_{ \pm}^{N K}
\end{array}
$$

from which we deduce the orthogonality conditions (31). (31) may also be checked directly by replacing the generators $\Lambda_{L}^{K}$ by their expression (29). Then $\Lambda_{L}^{K}$ generate a Lorentz algebra 
$\mathcal{L} \subset \mathcal{A}$ (Hopf algebra whose generators are subject to Lorentz group conditions (31)). Q.E.D.

The noncommutativity between the generators $\Lambda_{L}^{K}$ and the elements of $\mathcal{A}$ are given by

Theorem $(3,2)$ :

There exist functionals $F_{ \pm L}^{K}: \mathcal{A} \rightarrow C$ given by

$$
F_{ \pm L}^{K}=\frac{1}{Q}\left(\tilde{f}_{\mp \dot{\beta}}^{\dot{\alpha}} \bar{\sigma}_{L \dot{\alpha}}{ }^{\delta} \star f_{ \pm \delta}^{\gamma} \sigma_{\gamma}^{K} \dot{\beta}\right)
$$

satisfying:

a)

$$
\Lambda_{L}^{I}\left(F_{ \pm I}^{K} \star a\right)=\left(a \star F_{ \pm L}^{I}\right) \Lambda_{I}^{K}
$$

b)

$$
\left(F_{ \pm L}^{K}(a)\right)^{\star}=F_{ \pm L}^{K}\left(S\left(a^{\star}\right)\right)
$$

c)

$$
\begin{aligned}
F_{ \pm L}^{K}(a b) & =F_{ \pm L}^{I}(a) F_{ \pm I}^{K}(b) \\
F_{ \pm L}^{K}(\varepsilon(a)) & =\delta_{L}^{K} \varepsilon(a) \\
\left(F_{ \pm L}^{I} \star F_{ \pm I}^{K} \circ S\right)(a)=\left(F_{ \pm L}^{I} \circ S \star F_{ \pm I}^{K}\right)(a) & =\delta_{L}^{K} \varepsilon(a)
\end{aligned}
$$

d)

$$
\mathcal{R}_{K L}^{ \pm N M} G_{ \pm}^{K L}=G_{ \pm}^{N M} \quad, \quad \mathcal{R}_{K L}^{ \pm N M} G_{ \pm N M}=G_{ \pm K L}
$$

where $\mathcal{R}_{K L}^{ \pm N M}=F_{ \pm K}^{M}\left(\Lambda_{L}^{N}\right)$ satisfy the Yang-Baxter equations and the Hecke relations

$$
\left(\mathcal{R}^{ \pm}+a^{ \pm 2}\right)\left(\mathcal{R}^{ \pm}+a^{\mp 2}\right)\left(\mathcal{R}^{ \pm}-1\right)=0
$$

Proof: a) $X_{\alpha \dot{\beta}}$ are a right invariant basis of $\mathcal{A}$ - $\mathcal{M}$ bimodule transforming under the $\mathcal{L}$ algebra as (21). Then we can follow the Woronowicz formalism of the bicovariant bimodule [4] to state

$$
\begin{array}{r}
X_{\alpha \dot{\beta}} a=\left(a \star\left(f_{\mp \dot{\beta}}^{\dot{\rho}} \star f_{ \pm \alpha}^{\sigma}\right)\right) X_{\sigma \dot{\rho}}=f_{\mp \dot{\beta}} \dot{\rho}\left(a_{(1)}\right) f_{ \pm \alpha}^{\sigma}\left(a_{(2)}\right) a_{(3)} X_{\sigma \dot{\rho}}, \\
a X_{\alpha \dot{\beta}}=X_{\sigma \dot{\rho}}\left(a \star\left(f_{\mp \dot{\beta}}^{\dot{\rho}} \star f_{ \pm \alpha}^{\sigma}\right) \circ S\right)=X_{\sigma \dot{\rho}} f_{ \pm \alpha}^{\sigma}\left(S\left(a_{(1)}\right)\right) f_{\mp \dot{\beta}} \dot{\rho}\left(S\left(a_{(2)}\right)\right) a_{(3)}
\end{array}
$$

for any $a \in \mathcal{A}$. The convolution product of two functional is defined as $\left(f_{1} \star f_{2}\right)(a)=\left(f_{1} \otimes\right.$ $\left.f_{2}\right) \Delta(a)$. This choice of functionals is justified by the fact that if we apply the $\star$ involution on both sides of the first relation of (45) and we use the second relation with $a=a^{\star}$, we obtain

$$
a^{\star} X_{\beta \dot{\alpha}}=X_{\rho \dot{\sigma}}\left(f_{\mp \dot{\rho}}^{\dot{\rho}}\left(a_{(1)}\right)\right)^{\star}\left(f_{ \pm \alpha}^{\sigma}\left(a_{(2)}\right)\right)^{\star}\left(a_{(3)}\right)^{\star}=X_{\rho \dot{\sigma}} f_{ \pm \beta}^{\rho}\left(S\left(a_{(1)}^{\star}\right)\right) f_{\mp \dot{\alpha}}^{\dot{\sigma}}\left(S\left(a_{(2)}^{\star}\right)\right)\left(a_{(3)}\right)^{\star}
$$


which is consistent with (14). Now, the bicovariant bimodule formalism [4] can also be applyied to the $\mathcal{A}-\mathcal{M}$ bimodule to get

$$
M_{\alpha}^{\rho} M_{\dot{\beta}}^{\dot{\sigma}}\left(\left(f_{\mp \dot{\sigma}}^{\dot{\delta}} \star f_{ \pm \rho}^{\gamma}\right) \star a\right)=\left(a \star\left(f_{\mp \dot{\beta}}^{\dot{\sigma}} \star f_{ \pm \alpha}^{\rho}\right)\right) M_{\rho}^{\gamma} M_{\dot{\sigma}}^{\dot{\delta}}
$$

from which we deduce

$$
M_{\alpha}{ }^{\sigma} S^{-1}\left(M_{\dot{\mu}}^{\dot{\nu}}\right)\left(\tilde{f}_{\mp \dot{\beta}}^{\dot{\mu}} \star f_{ \pm \sigma}^{\gamma} \star a\right)=\left(a \star\left(\tilde{f}_{\mp \dot{\mu}}^{\dot{\mu}} \star f_{ \pm \alpha}{ }^{\sigma}\right) M_{\sigma}^{\gamma} S^{-1}\left(M_{\dot{\beta}}^{\dot{\mu}}\right)\right.
$$

where we have used (15). Note that this equation is a combination of the first equation of (9) and the second equation of (10).

Multiplying now both sides from the left by $\bar{\sigma}_{L \dot{\nu}}^{\alpha}$ and from right by $\sigma_{\gamma}^{K} \dot{\beta}$ and using the completeness relation $(27)$, we obtain

$$
\begin{aligned}
& \frac{1}{Q} \bar{\sigma}_{L \dot{\nu}}{ }^{\alpha} M_{\alpha}{ }^{\sigma} \sigma_{\sigma}^{I}{ }_{\sigma}^{\dot{\mu}} S^{-1}\left(M_{\dot{\mu}}^{\dot{ }}\right)\left(\left(\tilde{f}_{\mp \dot{\beta}}^{\dot{\tau}} \bar{\sigma}_{I \dot{\tau}}{ }^{\delta} \star f_{ \pm \delta}^{\gamma} \sigma_{\gamma}^{K} \dot{\beta}\right) \star a\right)= \\
& \left(a \star\left(\tilde{f}_{\mp \dot{\mu}}^{\dot{\nu}} \bar{\sigma}_{L \dot{\nu}}{ }^{\alpha} \star f_{ \pm \alpha} \sigma^{\sigma} \sigma_{\sigma}^{I} \dot{\mu}\right)\right) \frac{1}{Q} \bar{\sigma}_{I \dot{\tau}}{ }^{\delta} M_{\delta}^{\gamma} \sigma_{\gamma}^{K}{ }^{\dot{\beta}} S^{-1}\left(M_{\dot{\beta}}^{\dot{\tau}}\right)
\end{aligned}
$$

yielding

$$
\Lambda_{L}^{I}\left(F_{ \pm I}^{K} \star a\right)=\left(a \star F_{ \pm L}^{I}\right) \Lambda_{I}^{K}, \text { with } F_{ \pm L}^{K}=\frac{1}{Q}\left(\tilde{f}_{\mp \dot{\beta}}^{\dot{\alpha}} \bar{\sigma}_{L \dot{\alpha}}^{\delta} \star f_{ \pm \delta}^{\gamma} \sigma_{\gamma}^{K} \dot{\beta}\right)
$$

b) Using (14), (33) and (15), we get

$$
\begin{array}{r}
\left(F_{ \pm L}^{K}(a)\right)^{\star}=\frac{1}{Q}\left(G_{ \pm L N} \varepsilon_{\dot{\alpha} \dot{\rho}} \tilde{f}_{\mp \dot{\beta}}^{\dot{\alpha}}\left(a_{(1)}\right) \bar{\sigma}_{ \pm}^{N \dot{\rho} \delta} f_{ \pm \delta}^{\gamma}\left(a_{(2)}\right) \sigma_{\gamma \dot{\lambda}}^{K} \varepsilon^{\dot{\beta} \dot{\lambda}}\right)^{\star}= \\
\frac{1}{Q} \varepsilon^{\lambda \beta}\left(f_{ \pm \delta}^{\gamma}\left(a_{(2)}\right)\right)^{\star} \sigma_{\lambda \dot{\gamma}}^{K}\left(\tilde{f}_{\mp \dot{\beta}}^{\dot{\alpha}}\left(a_{(1)}\right)\right)^{\star} \bar{\sigma}_{ \pm}^{N \dot{\delta} \rho} G_{ \pm N L} \varepsilon_{\rho \alpha}= \\
\frac{1}{Q} \varepsilon^{\lambda \beta} f_{\mp \dot{\delta}}^{\dot{\gamma}}\left(S\left(a_{(2)}^{\star}\right)\right) \sigma_{\lambda \dot{\gamma}}^{K} \tilde{f}_{ \pm \beta}^{\alpha}\left(S\left(a_{(1)}^{\star}\right)\right) \varepsilon_{\dot{\nu} \dot{\mu}} \bar{\sigma}_{L}^{\dot{\mu} \rho} \varepsilon_{\alpha \rho} \varepsilon^{\dot{\nu} \dot{\delta}}= \\
\frac{1}{Q} \varepsilon^{\dot{\nu} \dot{\delta}} f_{\mp \dot{\delta}}^{\dot{\gamma}}\left(S\left(a_{(2)}^{\star}\right)\right) \varepsilon_{\dot{\gamma} \dot{\xi}} \sigma_{\lambda}^{K} \dot{\xi}_{\lambda}^{\lambda \beta} \tilde{f}_{ \pm \beta}^{\alpha}\left(S\left(a_{(1)}^{\star}\right)\right) \varepsilon_{\alpha \rho} \bar{\sigma}_{L \dot{\nu}}{ }^{\rho}= \\
\frac{1}{Q} \tilde{f}_{\mp \dot{\xi}}^{\dot{\nu}}\left(S\left(a_{(2)}^{\star}\right)\right) \bar{\sigma}_{L \dot{\nu}}^{\rho} f_{ \pm \rho}^{\lambda}\left(S\left(a_{(1)}^{\star}\right)\right) \sigma_{\lambda}^{K}{ }_{\lambda}^{\dot{\xi}}=F_{ \pm L}^{K}\left(S\left(a^{\star}\right)\right) .
\end{array}
$$

c) (40) is deduced directly from (6), (7) and the completeness relation (27) as

$$
\begin{array}{r}
F_{ \pm L}^{K}(a b)=\frac{1}{Q} \tilde{f}_{\mp \dot{\beta}}^{\dot{\alpha}}\left(a_{(1)} b_{(1)}\right) \bar{\sigma}_{L \dot{\alpha}}^{\delta} f_{ \pm \delta}^{\gamma}\left(a_{(2)} b_{(2)}\right) \sigma_{\gamma}^{K} \dot{\beta}= \\
\frac{1}{Q} \tilde{f}_{\mp \dot{\nu}}^{\dot{\alpha}}\left(a_{(1)}\right) \tilde{f}_{\mp \dot{\beta}}^{\dot{\nu}}\left(b_{(1)}\right) \bar{\sigma}_{L \dot{\alpha}}^{\delta} f_{ \pm \delta}^{\mu}\left(a_{(2)}\right) f_{ \pm \mu}^{\gamma}\left(b_{(2)}\right) \sigma_{\gamma}^{K} \dot{\beta}= \\
\frac{1}{Q} \tilde{f}_{\mp \dot{\rho}}^{\dot{\alpha}}\left(a_{(1)}\right) \tilde{f}_{\mp \dot{\beta}}^{\dot{\nu}}\left(b_{(1)}\right) \bar{\sigma}_{L \dot{\alpha}}^{\delta} f_{ \pm \delta}^{\mu}\left(a_{(2)}\right) f_{ \pm \tau}^{\gamma}\left(b_{(2)}\right) \sigma_{\gamma}^{K} \dot{\beta}_{\mu} \delta_{\mu}^{\tau} \delta_{\dot{\nu}}^{\dot{\rho}}= \\
\frac{1}{Q}\left(\tilde{f}_{\mp \dot{\rho}}^{\dot{\alpha}} \bar{\sigma}_{L \dot{\alpha}}^{\delta} \star f_{ \pm \delta}^{\mu} \sigma_{\mu}^{I} \dot{\rho}\right)(a) \frac{1}{Q}\left(\tilde{f}_{\mp \dot{\beta}}^{\dot{\nu}} \bar{\sigma}_{I \dot{\nu}}^{\tau} \star f_{ \pm \tau}^{\gamma} \sigma_{\gamma}^{K} \dot{\beta}\right)(b)=F_{ \pm L}^{I}(a) F_{ \pm I}^{I}(b) .
\end{array}
$$


We also have

$$
F_{ \pm L}^{K}(\varepsilon(a))=\frac{1}{Q} \tilde{f}_{\mp \dot{\beta}}^{\dot{\alpha}}\left(\varepsilon\left(a_{(1)}\right)\right) \bar{\sigma}_{L \dot{\alpha}}^{\delta} f_{ \pm \delta}^{\gamma}\left(\varepsilon\left(a_{(2)}\right)\right) \sigma_{\gamma}^{K} \dot{\beta}=\frac{1}{Q} \bar{\sigma}_{L \dot{\alpha}}{ }^{\delta} \sigma_{\delta}^{K} \dot{\alpha} \varepsilon(a)=\delta_{L}^{K} \varepsilon(a)
$$

where we have used (8). (42) can be deduced directly from (40) and (41).

d) Applying (37) on (29), we get

$$
\begin{gathered}
\mathcal{R}_{L K}^{ \pm N M} G_{ \pm}^{L K}=\frac{1}{Q}\left(\tilde{f}_{\mp \dot{\beta}}^{\dot{\tau}} \bar{\sigma}_{L \dot{\tau}}^{\delta} \star f_{ \pm \delta}^{\gamma} \sigma_{\gamma}^{M} \dot{\beta}\right)\left(\frac{1}{Q} \bar{\sigma}_{K \dot{\nu}}{ }^{\sigma} M_{\sigma}{ }^{\rho} \sigma_{\rho}^{N}{ }_{\rho}^{\dot{\lambda}} S^{-1}\left(M_{\dot{\lambda}}{ }^{\dot{\nu}}\right)\right) G_{ \pm}{ }^{L K}= \\
\frac{1}{Q^{2}} \tilde{f}_{\mp \dot{\beta}}^{\dot{\tau}}\left(M_{\sigma}^{\alpha} S^{-1}\left(M_{\dot{\mu}}^{\dot{\nu}}\right)\right) \bar{\sigma}_{L \dot{\tau}}{ }^{\delta} \bar{\sigma}_{K \dot{\nu}}{ }^{\sigma} f_{ \pm \delta}^{\gamma}\left(M_{\alpha}{ }^{\rho} S^{-1}\left(M_{\dot{\lambda}}^{\dot{\mu}}\right)\right) \sigma_{\gamma}^{M}{ }^{\dot{\beta}} \sigma_{\rho}^{N}{ }_{\rho}^{\dot{\lambda}} G_{ \pm}{ }^{L K}
\end{gathered}
$$

Using $G_{ \pm}^{L K} \bar{\sigma}_{L \dot{\tau}}{ }^{\delta} \bar{\sigma}_{K \dot{\nu}}{ }^{\sigma}=\bar{\sigma}_{L \dot{\tau}}{ }^{\delta} \bar{\sigma}_{ \pm \dot{\nu}}^{L}{ }^{\sigma}=R_{\dot{\nu} \mu}^{ \pm \alpha \dot{\beta}} \varepsilon^{\mu \sigma} \sigma_{\alpha \dot{\beta}}^{L} \bar{\sigma}_{L \dot{\tau}}^{\delta}=-Q\left(a^{ \pm \frac{1}{2}} \varepsilon^{\delta \nu} \varepsilon^{\tau \sigma}+a^{\mp \frac{1}{2}} \varepsilon^{\delta \tau} \varepsilon^{\sigma \nu}\right)$, obtained from (22), the completeness relation (27), (16) and the form of the $\mathrm{R}$ matrices, we get

$$
\begin{array}{r}
-\frac{1}{Q} \tilde{f}_{\mp \dot{\kappa}}^{\dot{\tau}}\left(M_{\sigma}^{\alpha}\right) \tilde{f}_{\mp \dot{\beta}}^{\dot{\kappa}}\left(S^{-1}\left(M_{\dot{\mu}}^{\dot{ }}\right)\right) f_{ \pm \delta}^{\xi}\left(M_{\alpha}{ }^{\rho}\right) f_{ \pm \xi}^{\gamma}\left(S^{-1}\left(M_{\dot{\lambda}}^{\dot{\mu}}\right)\right)\left(a^{ \pm \frac{1}{2}} \varepsilon^{\delta \nu} \varepsilon^{\tau \sigma}+a^{\mp \frac{1}{2}} \varepsilon^{\delta \tau} \varepsilon^{\sigma \nu}\right)= \\
-\frac{1}{Q} R_{\tau \sigma}^{\mp \alpha \kappa} R_{\kappa \nu}^{\mp \mu \beta} R_{\delta \alpha}^{ \pm \rho \xi} R_{\xi \mu}^{ \pm \lambda \gamma}\left(a^{ \pm \frac{1}{2}} \varepsilon^{\delta \nu} \varepsilon^{\tau \sigma}+a^{\mp \frac{1}{2}} \varepsilon^{\delta \tau} \varepsilon^{\sigma \nu}\right)
\end{array}
$$

where we have used (17). An explicit computation gives

$$
R_{\tau \sigma}^{\mp \alpha \kappa} R_{\kappa \nu}^{\mp \mu \beta} R_{\delta \alpha}^{ \pm \rho \xi} R_{\xi \mu}^{ \pm \lambda \gamma} \varepsilon^{\delta \nu} \varepsilon^{\tau \sigma}=\varepsilon^{\rho \beta} \varepsilon^{\lambda \gamma} \text { and } R_{\tau \sigma}^{\mp \alpha \kappa} R_{\kappa \nu}^{\mp \mu \beta} R_{\delta \alpha}^{ \pm \rho \xi} R_{\xi \mu}^{ \pm \lambda \gamma} \varepsilon^{\delta \tau} \varepsilon^{\sigma \nu}=\varepsilon^{\rho \lambda} \varepsilon^{\gamma \beta}
$$

from which we deduce

$$
\begin{aligned}
& \mathcal{R}_{L K}^{ \pm N M} G^{ \pm L K}=- \frac{1}{Q}\left(a^{ \pm \frac{1}{2}} \varepsilon^{\rho \beta} \varepsilon^{\lambda \gamma}+a^{\mp \frac{1}{2}} \varepsilon^{\rho \lambda} \varepsilon^{\gamma \beta}\right) \sigma_{\gamma}^{M} \dot{\beta} \sigma_{\rho}^{N \dot{\lambda}}= \\
& \frac{1}{Q}\left(a^{ \pm \frac{1}{2}} \varepsilon^{\rho \beta} \varepsilon_{\dot{\gamma} \dot{\lambda}}-a^{\mp \frac{1}{2}} \varepsilon^{\rho \lambda} \varepsilon^{\gamma \beta}\right) \sigma_{\gamma}^{M} \dot{\beta} \sigma_{\rho}^{N \dot{\lambda}}= \\
& \frac{1}{Q}\left(a^{ \pm \frac{1}{2}} \sigma_{\dot{\gamma}}^{N \beta} \sigma_{\gamma}^{M} \dot{\beta}-a^{\mp \frac{1}{2}} \sigma^{N \lambda \dot{\lambda}} \sigma^{M \beta \dot{\beta}}\right)=G_{ \pm}^{N M}
\end{aligned}
$$

where we have used (16) and (25). A similar calculation gives

$$
\mathcal{R}_{L K}^{ \pm N M} G_{ \pm N M}=\frac{1}{Q}\left(a^{\mp \frac{1}{2}} \bar{\sigma}_{L \delta}^{\dot{\beta}} \bar{\sigma}_{K \dot{\delta}}^{\beta}-a^{ \pm \frac{1}{2}} \bar{\sigma}_{L \dot{\beta} \beta} \bar{\sigma}_{K \dot{\delta} \delta}\right)=G_{ \pm L K}
$$

The Yang-Baxter equations may be obtained by applying $F_{ \pm L}^{K}$ on both sides of (38) for $a=\Lambda_{N}^{M}$ and then using (40). Using the Hecke relations of the $\mathrm{R}$ matrices, we obtain after a explicit straightforward calculation

$$
\begin{gathered}
\mathcal{R}_{I J}^{ \pm N M} \mathcal{R}_{L K}^{ \pm I J}=\left(2-a^{ \pm 2}-a^{\mp 2}\right) \mathcal{R}_{L K}^{ \pm N M}+\delta_{L}^{N} \delta_{K}^{M}+ \\
\frac{1}{Q^{2}} a^{\mp 2}\left(1-a^{ \pm 2}\right) R_{\delta \alpha}^{ \pm \rho \xi} R_{\xi \kappa}^{ \pm \lambda \gamma} R_{\tau \sigma}^{\mp \alpha \kappa} \sigma_{\gamma}^{M}{ }_{\gamma}^{\dot{\beta}} \sigma_{\rho}^{N} \dot{\lambda}_{\bar{\sigma}_{L}{ }_{L \bar{\tau}} \bar{\sigma}_{K \dot{\beta}}^{\sigma}+}+
\end{gathered}
$$




$$
\begin{aligned}
& \frac{1}{Q^{2}} a^{ \pm 2}\left(1-a^{\mp 2}\right) R_{\xi \nu}^{\mp \mu \beta} R_{\alpha \mu}^{ \pm \lambda \gamma} R_{\tau \sigma}^{\mp \alpha \xi} \sigma_{\gamma}^{M} \dot{\beta}_{\rho}^{N} \dot{\lambda}_{\bar{\sigma}_{L \dot{\tau}} \rho} \bar{\sigma}_{K \dot{\nu}}^{\sigma}
\end{aligned}
$$

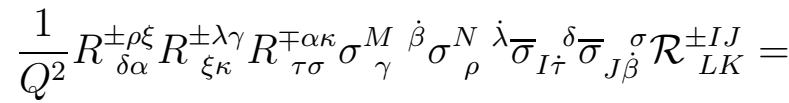

$$
\begin{aligned}
& \left(1-a^{ \pm 2}\right) \mathcal{R}_{L K}^{ \pm N M}+\frac{a^{ \pm 2}}{Q^{2}} R_{\xi \nu}^{\mp \mu \beta} R_{\kappa \mu}^{ \pm \lambda \gamma} R_{\tau \sigma}^{\mp \kappa \xi} \sigma_{\gamma}^{M} \dot{\beta}_{\rho} \sigma_{\rho}^{N} \dot{\lambda}_{L \dot{\tau}}^{\rho} \bar{\sigma}_{K \dot{\nu}}^{\sigma} \quad \text { and }
\end{aligned}
$$

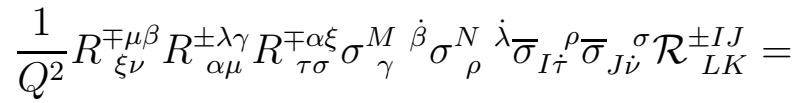

$$
\begin{aligned}
& \left(1-a^{\mp 2}\right) \mathcal{R}_{L K}^{ \pm N M}+\frac{a^{\mp 2}}{Q^{2}} R_{\xi \kappa}^{ \pm \delta \alpha} R_{\alpha \mu}^{ \pm \lambda \gamma} R_{\tau \sigma}^{\mp \kappa \mu} \sigma_{\gamma}^{M} \dot{\beta}_{\rho}^{N} \dot{\delta}_{\bar{\sigma}_{L \dot{\tau}}}^{\xi} \bar{\sigma}_{K \dot{\beta}}{ }_{\sigma}
\end{aligned}
$$

leading to (44). Q.E.D.

As for the $\mathcal{A}-\Gamma$ bimodule, the existence of two functionals $F_{ \pm L}^{K}$ leads to two right invariant basis $X_{ \pm I}$ of the $\mathcal{A}-\mathcal{M}$ bimodule satisfying

$$
\begin{array}{rlrl}
X_{ \pm L} a=\left(a \star F_{ \pm L}^{K}\right) X_{ \pm K} & , & X_{ \pm}^{( \pm) L} a=\left(a \star \tilde{F}_{ \pm K}^{( \pm) L}\right) X_{ \pm}^{( \pm) K} \\
a X_{ \pm L}=X_{ \pm K}\left(a \star F_{ \pm L}^{K} \circ S\right) & , \quad a X_{ \pm}^{( \pm) L}=X_{ \pm}^{( \pm) K}\left(a \star \tilde{F}_{ \pm K}^{( \pm)} L \circ S\right)
\end{array}
$$

for any $a \in \mathcal{A}$. The indices are raised and lowered by using the Minkowsky metric as $X_{L} G_{ \pm}^{L K}=$ $X^{( \pm) K}$. Following the same formalism applyied to the $\mathcal{A}-\Gamma$ bimodule, we may show that

$$
F_{ \pm L}^{K}=G_{ \pm}^{K M} \tilde{F}_{ \pm M}^{( \pm)}{ }^{N} G_{ \pm N L} \text { and } \tilde{F}_{ \pm L}^{( \pm)} K \circ S=F_{ \pm L}^{K}
$$

implying

$$
G_{ \pm}^{M N} F_{ \pm N}^{L} \star F_{ \pm M}^{K}(a)=G_{ \pm}^{K L} \varepsilon(a) \text { and } G_{ \pm K L} F_{ \pm N}^{L} \star F_{ \pm M}^{K}(a)=G_{ \pm M N} \varepsilon(a)
$$

for any $a \in \mathcal{A}$.

\section{Quantum Minkowsky space}

We consider the elements $X_{I}$ of right invariant basis of the $\mathcal{A}$ - $\mathcal{M}$ bimodule as coordinates which span the Minkowsky space $\mathcal{M}$ over the field $\mathrm{R}$. Then Minkowski space is equipped either with coordinates $X_{+I}$ and metric $G_{+I J}$ or with coordinates $X_{-I}$ and metric $G_{-I J}$. (32) shows that $\Delta_{L}: \mathcal{M} \rightarrow \mathcal{L} \otimes \mathcal{M}$ is a corepresentation of $\mathcal{L}$ in the vector space $\mathcal{M}$. In fact, from (32), (34) and the action of the counity on the generators of $\mathcal{L}$, it is easy to verify $\left(i d \otimes \Delta_{L}\right) \Delta_{L}=(\Delta \otimes i d) \Delta_{L}$ and $(\varepsilon \otimes i d) \Delta_{L}=i d$.

In the following we assume that the coordinates $X_{ \pm I}$ commute, in the quantum sens, with them selves and with the elements (spinors) of the right invariant basis of $\mathcal{A}-\Gamma$ bimodule. To carry this quantum symmetrization, we consider the bicovariant bimodule automorphism $\sigma$ [4] 
such that for any $a, b \in \mathcal{A}$ and the left invariant element $\tilde{X}_{ \pm I}=S\left(\Lambda_{I}^{J}\right) X_{ \pm J}, \tilde{\theta}_{ \pm \alpha}=S\left(M_{\alpha}^{\beta}\right) \theta_{ \pm \beta}$ or $\tilde{\theta}_{ \pm \dot{\alpha}}=S\left(M_{\dot{\alpha}}^{\dot{\beta}}\right) \theta_{ \pm \dot{\beta}}$, we have

$$
\begin{array}{r}
\sigma\left(\tilde{X}_{(a) L} \otimes X_{(b) K}\right)=X_{(b) K} \otimes \tilde{X}_{(a) L}=X_{(b) K} \otimes S\left(\Lambda_{L}^{N}\right) X_{(a) N}= \\
S\left(\Lambda_{L}^{N}\right) \sigma\left(X_{(a) N} \otimes X_{(b) K}\right)=F_{(b) K}{ }^{Q}\left(S\left(\Lambda_{P}^{N}\right)\right) S\left(\Lambda_{L}^{P}\right)\left(X_{(b) Q} \otimes X_{(a) N}\right)
\end{array}
$$

where $a, b= \pm$ or $\mp$. From the later equation we deduce

$$
\sigma\left(X_{(a) L} \otimes X_{(b) K}\right)=F_{(b) K}^{N}\left(S\left(\Lambda_{L}^{M}\right)\right)\left(X_{(b) N} \otimes X_{(a) M}\right) .
$$

For the spinors, it suffices to replace $\Lambda_{L}^{K}$ by $M_{\alpha}{ }^{\beta}$ or $M_{\dot{\alpha}}^{\dot{\beta}}$ and $X_{(a) I}$ by $\theta_{(a) \alpha}$ or $\theta_{(a) \dot{\alpha}}$. The symmetrization of the product is defined as

$$
\begin{gathered}
X_{(a) L} X_{(b) K}=F_{(b) K}^{N}\left(S\left(\Lambda_{L}^{M}\right)\right)\left(X_{(b) N} X_{(a) M}\right) \\
\theta_{(a) \alpha} X_{(b) K}=F_{(b) K}^{N}\left(S\left(M_{\alpha}^{\beta}\right)\right)\left(X_{(b) N} \theta_{(a) \beta}\right) \quad
\end{gathered}
$$

From this, we state

Theorem $(4,1)$ :

The norm of $X_{ \pm}, G_{ \pm}^{I J} X_{ \pm I} X_{ \pm J}$ is invariant and central.

Proof.: By construction, $X_{ \pm I}$ is right invariant. As a consequence of the orthogonality condition of the generators of $\mathcal{L}$, we may easily see from the transformations of $X_{ \pm I}(34)$ that $G_{ \pm}^{I J} X_{ \pm I} X_{ \pm J}$ is left invariant. From (47) and (48), we get, for any $a \in \mathcal{A}$,

$$
\begin{array}{r}
G_{ \pm}^{I J} X_{ \pm I} X_{ \pm J} a=\left(a \star G_{ \pm}^{I J}\left(F_{ \pm J}^{L} \star F_{ \pm I}^{K}\right)\right) X_{ \pm K} X_{ \pm L}= \\
(a \star \varepsilon) G_{ \pm}{ }^{K L} X_{ \pm K} X_{ \pm L}=a G_{ \pm}{ }^{K L} X_{ \pm K} X_{ \pm L}
\end{array}
$$

which shows that the norm commutes with any $a \in \mathcal{A}$.

From (48) and (49), we obain

$$
\begin{array}{r}
X_{(a) P} G_{ \pm}^{I J} X_{ \pm I} X_{ \pm J}=G_{ \pm}^{I J} F_{ \pm I}^{N}\left(S\left(\Lambda_{P}^{M}\right)\right) F_{ \pm J}^{K}\left(S\left(\Lambda_{M}^{L}\right)\right) X_{ \pm N} X_{ \pm K} X_{(a) L}= \\
G_{ \pm}^{I J}\left(F_{ \pm J}^{K} \star F_{ \pm I}^{N}\right)\left(S\left(\Lambda_{P}^{L}\right)\right) X_{ \pm N} X_{ \pm K} X_{(a) L}=G_{ \pm}{ }^{N K} X_{ \pm N} X_{ \pm K} X_{(a) P}
\end{array}
$$

The same results may be obtained by replacing $X_{(a) P}$ by $\theta_{(a) \alpha}$ or $\theta_{(a) \dot{\alpha}}$ which show that the norm also commutes with the quantum coordinates and the spinors. Q.E.D.

Remark $(4,1)$ :

- $G_{ \pm}^{L K} X_{ \pm L} X_{ \pm K}$ is biinvariant and real. Since it commutes with everything, it is of the form $\lambda I_{\mathcal{L}}$ with $\lambda$ is a real number.

- The quantities $G_{ \pm}^{I J} X_{\mp I} X_{\mp J}$ and $G_{ \pm}^{I J} X_{\mp I} X_{ \pm J}$ are biinvariant but not central.

Acknowledgments. I am grateful to M. Dubois-Violette for his kind interest and hepful suggestion. I also thank N. Touhami for valuable discussions and S. Balaska for his help which 
allowed me to have results from the computer MAPLE program.

\section{References:}

1)A. Connes, Publ, IHES 62(1986)257, Noncommutative Geometry, Academic Press, inc. (1994).

2)R. Coquereaux, G. Esposito-Farese and G. Vaillant, Nucl. Phys. B353(1991) 689.

3)M. Dubois-Violette, R. Kerner and J. Madore, Class. Quant. Grav. 6(1989)1709, J. Math. Phys. 31(1990)323.

4)S. L. Woronowicz, Commun. Math. Phys. 122(1989)125.

5)P. Podles and S.L. Woronowicz, Commun. Math. Phys. 130(1990)381.

6)U. Carow-Watamura, M. Schlieker, M. Scholl and S. Watamura, Z. Phys. C- partcles and fields 48(1990)159, Int. J. Mod. Phys. A6(1991)3081.

7)O. Ogiesvestsky, W. B. Schmidke, J. Wess and B. Zumino, Commun. Math. Phys. 150(1992)495.

8)P. Podles and S. L. Woronowicz, Commun. Math. Phys. 178(1996)61, Commun. Math. Phys. 185(1997)325.

9)M. Dubois-Violette and G. Launer, Phys. Lett. B245(1990)175.

10)M. Lagraa, Int. J. Mod. Phys. A11(1996)699.

11)A. B. Hammou and M. Lagraa, J. Math. Phys. 38(1997)4462. 